\title{
Interfaces
}

\section{Les métamorphoses du ciel : espaces et formats dans la poésie spatialiste}

\section{Marianne Simon-Oikawa}

\section{(2) OpenEdition}

1 Journals

Édition électronique

URL : https://journals.openedition.org/interfaces/2009

DOI : 10.4000/interfaces.2009

ISSN : 2647-6754

Éditeur :

Université de Bourgogne, Université de Paris, College of the Holy Cross

\section{Édition imprimée}

Date de publication : 12 juillet 2021

ISSN : 1164-6225

\section{Référence électronique}

Marianne Simon-Oikawa, "Les métamorphoses du ciel : espaces et formats dans la poésie spatialiste », Interfaces [En ligne], 45 | 2021, mis en ligne le 12 juillet 2021, consulté le 15 septembre 2021. URL : http://journals.openedition.org/interfaces/2009; DOI : https://doi.org/10.4000/interfaces. 2009

Ce document a été généré automatiquement le 15 septembre 2021.

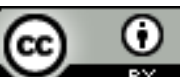

Les contenus de la revue Interfaces sont mis à disposition selon les termes de la Licence Creative Commons Attribution 4.0 International. 


\title{
Les métamorphoses du ciel : espaces et formats dans la poésie spatialiste
}

\author{
Marianne Simon-Oikawa
}

1 Figures importantes des avant-gardes et inventeurs du "spatialisme " au début des années 1960, Ilse Garnier (1927-2020) et Pierre Garnier (1928-2014) sont surtout connus pour leurs créations dans le domaine de la poésie visuelle et de la poésie sonore ${ }^{1}$. L'adjectif "spatialiste", qui distingue leur mouvement d'autres poésies de la même époque comme la poésie concrète ou la poesia visiva en Italie, renvoie à une notion qui est à considérer dans toutes ses acceptions : chez eux l'espace est à la fois cosmique, géographique, mental, plastique, matériel. Dans leurs œuvres visuelles, qui nous intéresseront ici, il s'incarne d'abord dans le support du poème: le poète artisan dispose sur la page des signes (mots, lettres, signes de ponctuation, chiffres dans les années 1960 et 1970, auxquels s'ajoutent dans les décennies suivantes des éléments graphiques et plastiques de nature plus variée) qu'il fait tenir ensemble par de savants jeux d'équilibre et de tension. L'espace du poème peut lui-même prendre plusieurs formes. Feuilles sous pochette, livres reliés, projection de diapositives et même installations éphémères, l'inventivité d'Ilse et Pierre Garnier fut remarquable aussi bien dans l'expérimentation de supports peu habituels en poésie que dans la réinvention d'autres plus classiques.

2 Les chercheurs ont souvent souligné l'importance de la page et du blanc dans la poésie spatialiste, et plusieurs recueils remarquables par leur dispositif non conventionnel ont fait l'objet d'une attention plus particulière, notamment les livres à quatre mains cosignés par les deux poètes ou encore les leporellos ${ }^{2}$. Le format en tant que tel n'a toutefois jamais été utilisé comme fil directeur d'une enquête sur l'ensemble de leurs œuvres respectives. La définition qu'en donne David Zerbib comme «construction médiatrice » (16) induisant des manières spécifiques de voir, de percevoir, de faire et de connaître, invite au contraire à se saisir de cette notion pour relire la production spatialiste et en penser les caractéristiques formelles de manière dynamique. Pour Ilse et Pierre Garnier, les supports ne sont pas simplement des objets dotés de certaines dimensions. Ils constituent autant d'occasions de jouer avec les normes, de faire sortir 
le poème de la page, de renouveler les formes du livre, et de faire circuler leurs créations d'un support à un autre, bref d'inventer de nouveaux espaces pour une nouvelle poésie.

\section{Sortir de la page}

3 Le « site naturel » du poème concret est la page, rappelle Anne Mœglin-Delcroix (81). C'est donc par cette dernière qu'il convient de commencer l'enquête. Chez Ilse et Pierre Garnier, la page est souvent une simple feuille de papier A4. C'est dans ce format que les poètes ont composé nombre de leurs manuscrits et que plusieurs de leurs recueils ont été imprimés, même s'il est loin d'être le seul : d'autres œuvres furent réalisées directement sur des papiers de grands formats, voire sur des supports comme la toile ou le plexiglas (P.Garnier, L'isola). Précisons d'emblée cependant que dans la perspective spatialiste la page même la plus banale n'est pas un espace neutre : elle est en effet pensée en relation avec le cosmos.

4 Fasciné par le ciel, Pierre Garnier formule à de nombreuses reprises l'idée d'un rapport entre la page et l'espace cosmique. À des déclarations comme «Identification de la page à l'espace, au ciel » (Édeline 1981 82) ou "Que nos mots, eux aussi, rejoignent l'espace cosmique - les mots-étoiles sur la page blanche» (P. Garnier, «Manifeste pour une poésie nouvelle, visuelle et phonique » 2), font écho des poèmes dans lesquels des mots écrits sur une feuille ou un tableau d'école sont comparés à des étoiles, comme dans cette scène où Pierre Garnier met en scène l'oncle jardinier dans une de ses activités familières :

il lui arrivait

de prendre une carte du ciel,

de marquer sur un Canson

des points à la place des étoiles,

d'écrire là des mots -

" un beau poème » disait-il ${ }^{3}$ (P. Garnier, Une mort toujours enceinte 30)

5 Cette représentation de la composition poétique renvoie à plusieurs caractéristiques de la poésie spatialiste : le papier Canson indique la proximité entre le poème visuel et les arts plastiques; la présence d'étoiles signale l'importance de la "constellation », principe de composition qui consiste à disposer des signes écrits sur la page à la manière de corps célestes dans le ciel ${ }^{4}$; la disposition des mots en différents points de la page souligne le refus de la syntaxe et le désir de les traiter isolément en tant qu'objets autonomes. On peut y déceler aussi la trace d'une réflexion sur le format : à l'origine était le ciel, et le poème a directement à voir avec un changement d'échelle.

\section{Miniaturiser}

La miniaturisation intéressa beaucoup Ilse et Pierre Garnier. Ils aimaient les enluminures décorant les manuscrits médiévaux, ainsi que les faïences anciennes qui leur semblaient contenir un univers entier. Les signes avec lesquels ils composent leurs poèmes sont pour eux des «microsystèmes" (P. Garnier, «Microstructures et microformats »a 28), et la création poétique tout entière est faite de «microsensibilités, microsentiments, microactions, microréactions» (P. Garnier, «Position 4 du Spatialisme » 209). La micropoésie a à voir avec le minimalisme des moyens plus qu'avec les seules dimensions des signes ou des poèmes eux-mêmes; elle 
constitue un principe esthétique et non une instruction d'ordre technique. On pourra donc en trouver la trace sur toutes sortes de formats, y compris de grandes dimensions.

7 Reste que le choix de petits formats (proches du B6 ou inférieurs) pour certains recueils s'inscrit directement dans la continuité de cette réduction des matériaux poétiques. Pierre Garnier le précise explicitement: «les poètes spatialistes limitent volontairement les formats de certains de leurs poèmes : ces "miniatures" s'adressent au lecteur seul. Leur surface est mesurée de telle façon que l'œil perçoive sans se déplacer les champs d'intensité » (P. Garnier, «Microstructures et microformats » b 87). Plusieurs livres furent publiés dans des collections de petite taille (format A6), comme Depuis qu'il n'y a plus d'abeilles, la poésie a quitté le village, A vécu la disparition des bouvreuils, ou encore Adolescence. Certains titres sont aussi très clairs, depuis I microcosmique, variation sur la lettre i tapée à la machine dans une démarche caractéristique de la production spatialiste des années 1960, jusqu'à des recueils beaucoup plus tardifs de la fin des années 1990 et des années 2000 comme Poèmes sous microscope ou encore Nanopoèmes, qui reposent sur la combinaison de dessins et de mots.

Une autre forme de miniaturisation de l'espace, plus discrète, est le compartimentage : la page, quelles que soient ses dimensions, est divisée en plusieurs cases comprenant chacune un poème. Cette pratique, qui traverse toute l'œuvre de Pierre Garnier, ne consiste pas seulement à déterminer de petits espaces : elle doit être comprise comme un moyen de circonscrire de manière visuelle le territoire adapté à chaque œuvre. "Définir un cadre, c'est définir une fonction et un mode opératoire ", rappelle Emmanuël Souchier (23). Dans le cas de Pierre Garnier, c'est déterminer le rapport entre la taille des signes écrits et l'importance des blancs qui se trouvent à l'intérieur, mais aussi jouer avec le texte placé à l'extérieur du cadre et qui l'accompagne. Dès le numéro de décembre 1966 de la revue vers-univers de Frans Vanderlinde, les «minipoems » sont reproduits deux à trois par page, encadrés chacun dans une case plus petite (Fig. 1). Ils l'étaient déjà dans le premier état du manuscrit, ce qui suggère que l'équilibre de l'ensemble, celui de chaque poème mais aussi celui de la page tout entière, fut pensé dès le début par Pierre Garnier. 
Figure 1. Pierre Garnier. « minipoems ». vers-univers (décembre 1966), p. 9.

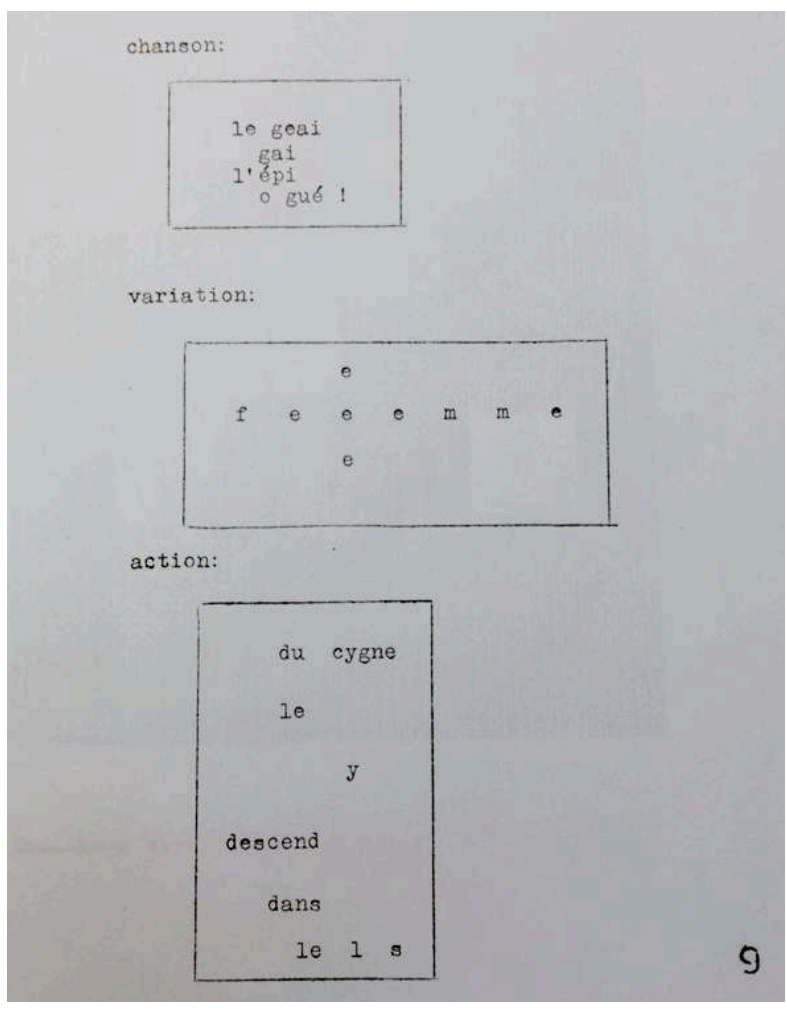

9 En 1969, les poèmes que Pierre Garnier envoie au poète japonais Seiichi Niikuni sous le titre Micropoèmes se présentent chacun dans un quart de feuille A4 délimité au stylo (P. et I. Garnier, Japon vol. I 195-206) (Fig. 2). Complétés par Niikuni à l'aide de caractères japonais, ils furent publiés, un par page, dans le $\mathrm{n}^{\circ} 4$ de la revue ASA ( $21 \mathrm{x}$ $15 \mathrm{~cm}$ ) en 1970 (P. et I. Garnier, Japon vol. I 99-104). À partir des années 1980, Pierre Garnier utilisera souvent un cadre tracé à la main et de taille variable pour des poèmes composés de texte et de dessins, ainsi que pour des bandes dessinées, le texte étant parfois inclus dans la case, parfois non. Les deux cas se présentent d'ailleurs parfois à l'intérieur du même livre (voir par exemple le recueil de poèmes 16 Gedichte in einem Quadrat [16 poèmes dans un carré] et la bande dessinée Tintins Reise in die Poesie [Le voyage de Tintin en poésie]). 
Figure 2. Envoi de Pierre Garnier à Seiichi Nikuni (1969).

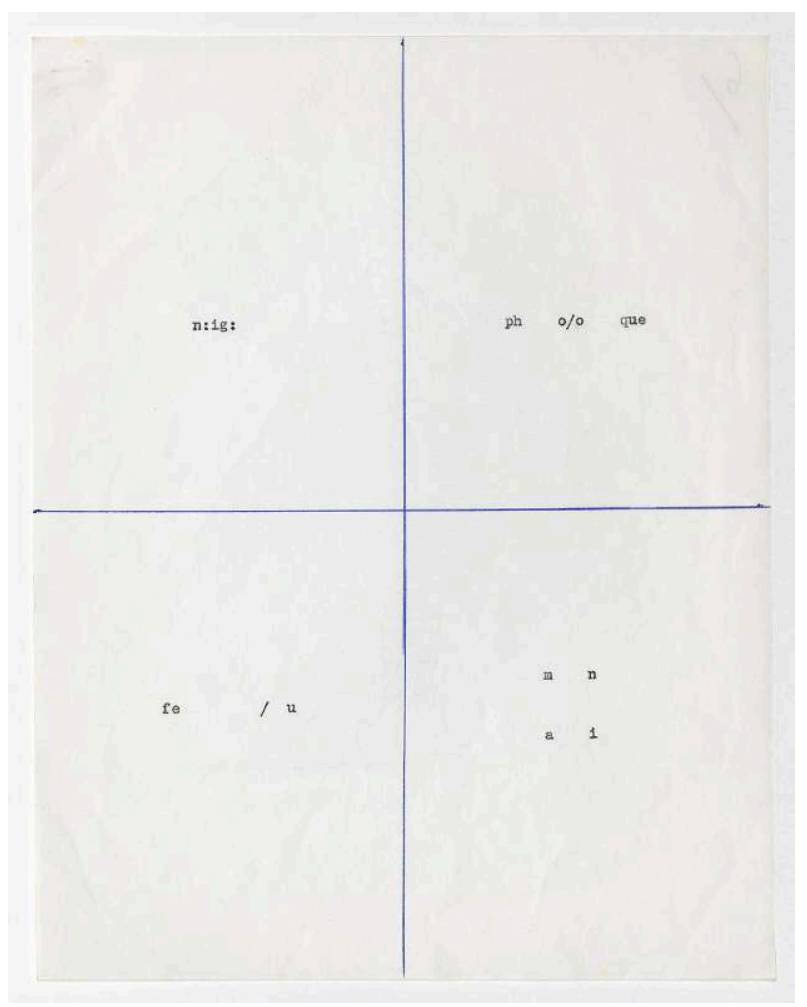

(c) Violette Garnier et Musashino Art University Museum \& Library, Japan.

\section{Déborder}

Si la page est un microcosme et le poème une combinaison de "microsystèmes ", les poèmes spatialistes ne tiennent pas tous sur de petits formats. C'est que le rapport de la page au ciel n'est pas simplement un rapport d'échelle. C'est aussi un rapport d'énergie. De même que les étoiles dans le ciel sont organisées entre elles en vertu de subtils rapports d'attraction, de même pour les poètes spatialistes les signes sur la page sont unis par des relations qui peuvent être d'ordre spatial, formel, ou sémantique. Le spatialisme pense ces rapports à l'aide de métaphores énergétiques, en termes de «rayonnement", de "vibration», ou encore de "dilatation». Du point de vue du lecteur-spectateur placé devant le poème, chaque signe possède une puissance propre, née de sa matérialité (police, graisse), et « c'est justement entre cette unité-mot et cette autre unité-mot, entre ce centre d'énergie et cet autre centre d'énergie que s'établit le réseau de forces et de tensions constituant le poème visuel » (I. Garnier, « Fin du monde de l'expression» 44). Les poèmes en apparence les plus statiques qui évoquent un paysage, comme « Hiver " par exemple, un des premiers poèmes spatiaux publiés par Pierre Garnier, ne font pas exception (Fig. 3). L'immobilité des signes sur le support est compensée par l'activité de la perception: le regard passe d'un mot à un autre, construit mentalement la relation qui les unit, passe à un troisième, etc. (Lengellé 243-45). 
Figure 3. Pierre Garnier. « Hiver ». Les Lettres 29 (1 ${ }^{\mathrm{er}}$ trimestre 1963).

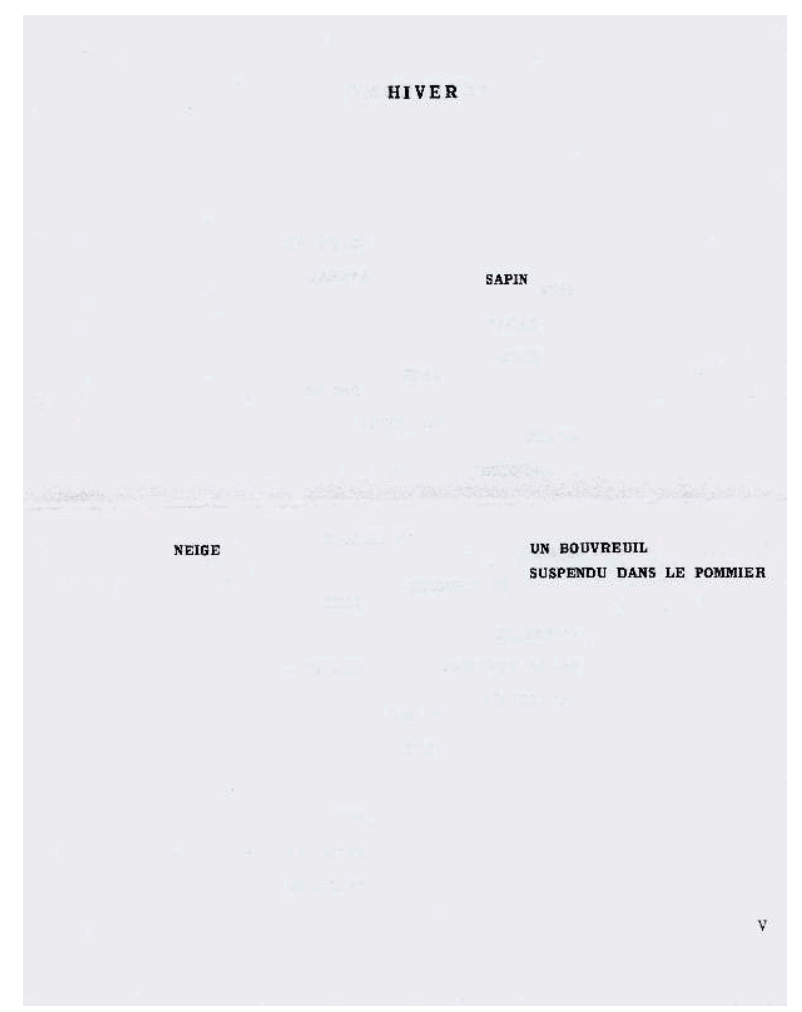

11 Une telle conception du poème n'est pas sans conséquence sur la question des formats. Car si la page est un support sur lequel les signes écrits irradient, au point qu'il faille « considérer la lecture comme la perception de vibrations lumineuses » (P. Garnier, «Position 4 du Spatialisme » 212), il est évident que ses limites matérielles ne sauraient enfermer le poème.

Il faut que le poème visuel déborde la page. Je m'explique : le poème jusqu'alors reste sagement en équilibre sur sa page. Le poème visuel, au contraire, tend sans cesse à répandre ses mots. Voyez, ils arrivent librement jusqu'au bord, ils tentent de rejoindre l'univers, ils vibrent, ils vont se perdre dans les espaces. (P. Garnier, « Deuxième manifeste pour une poésie visuelle»17)

12 Ilse Garnier fait du passage un des principes de son esthétique (Maunet-Salliet). Dans un poème de "Passages ", la troisième partie de son recueil Poème $d u i$, la flèche orientée vers le haut invite le lecteur à suivre une ligne imaginaire au-delà du support, et à transgresser au sens propre ses limites. Les éléments graphiques semblent littéralement sortir de la page (Fig. 4). 
Figure 4. Ilse Garnier. Poème du i, Poème spatial. Paris : André Silvaire, 1981.

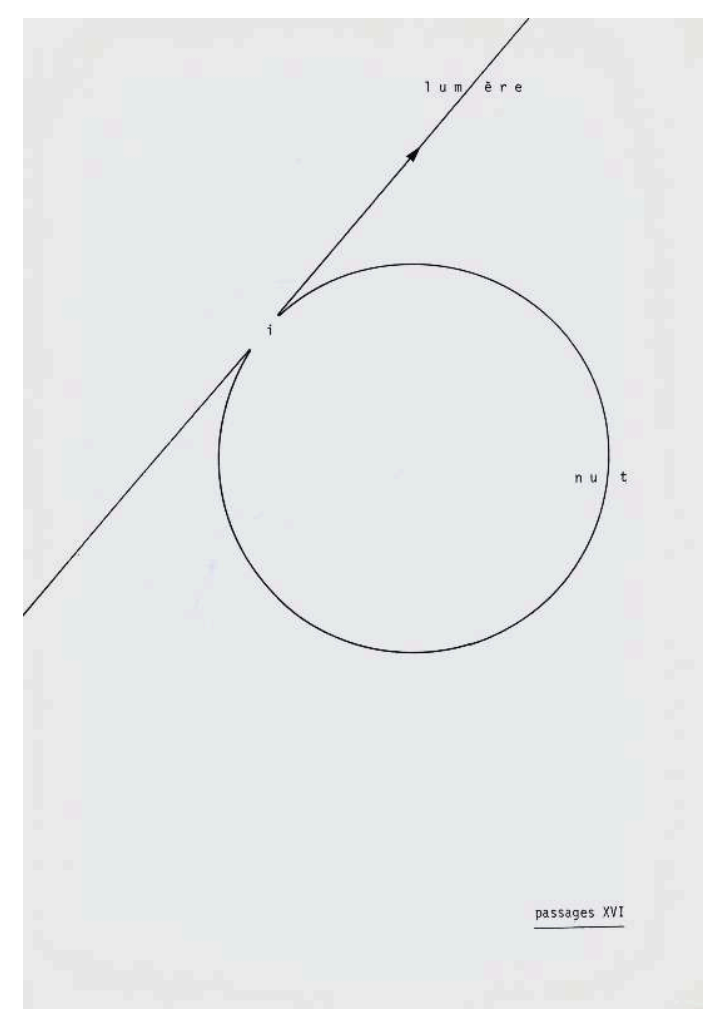

\section{De la page au mur}

13 Pierre et Ilse Garnier n'entendent pas se contenter de la feuille de papier. Ils veulent « inscrire le poème sur des murs, sur des pierres, sur des vitres, sur du sable figé, sur du papier d'emballage, sur de vieux sacs» (P. Garnier, "Deuxième manifeste pour une poésie visuelle » 19). Ils espèrent aussi «faire entrer la poésie dans les villes et dans les demeures ", et ébauchent une liste de supports possibles :

l'affiche, les poèmes visuels en céramique, des calendriers faits de poèmes visuels, un poème visuel se poursuivant, se complétant, se détruisant de chambre en chambre, des poèmes visuels mobiles en néon, des poèmes visuels sur cartes postales, des boîtes où seront enfermés quelques mots, des tapisseries - enfin, et peut-être surtout, le cinéma. (20)

Les poètes spatialistes ne réaliseront pas de poèmes en néon, mais ils s'intéresseront à l'architecture. L'idée de quitter la page et d'investir des lieux publics est alors en pleine maturation au sein des avant-gardes. En 1966 Ian Hamilton Finlay et sa femme Sue s'installeront au sud d'Édimbourg et commenceront à créer Little Sparta, leur immense jardin parsemé d'inscriptions sculptées; en 1967 Alain Arias-Misson réalisera son premier "public poem », en déployant les lettres du mot Vietnam dans une rue de Bruxelles.

La création spatialiste la plus aboutie dans le domaine architectural est un recueil de poèmes publié en 1965 et intitulé Prototypes. Textes pour une architecture (Fontana; Simon-Oikawa, "Esthétique de la 'poésie habitable'»). S'appuyant sur une analogie entre poésie et architecture ( en réalité architecture et poésie sont faites des mêmes éléments : points, lignes qui déterminent une surface, dimensions déterminant un volume $^{5} »$ ), Ilse et Pierre réalisent 23 poèmes spatiaux (22 originaux plus 1 repris de 
Poèmes mécaniques publié quelques mois plus tôt), dans l'espoir que des architectes s'en saisiront pour les inscrire sur "une surface ou un volume ", les déployer dans un lieu sous la forme d'un " continuum d'espace en espace », voire les " projeter sur un plan » sous la forme d'un film ou de diapositives (préface, n.p.). Un des poèmes est ainsi explicitement destiné à un vitrail, d'autres à un cinéma (Fig. 5), une morgue ou une église, et les poètes envisageaient même des poèmes pour une fenêtre, une porte, un cirque, une piscine, un cimetière, un mur d'église, un mur étroit d'école, un mur de maison de culture, un stade ou encore une gare. En 1972, Pierre Garnier envoya à Seiichi Niikuni un ensemble de quatre poèmes intitulé "Poèmes pour tapisseries" (Fig. 6), et un autre de six poèmes intitulé Cimetière spatialiste (P. et I. Garnier, Japon 114-26).

Figure 5. Ilse et Pierre Garnier. Prototypes. Poèmes pour une architecture. Paris : André Silvaire, 1965.

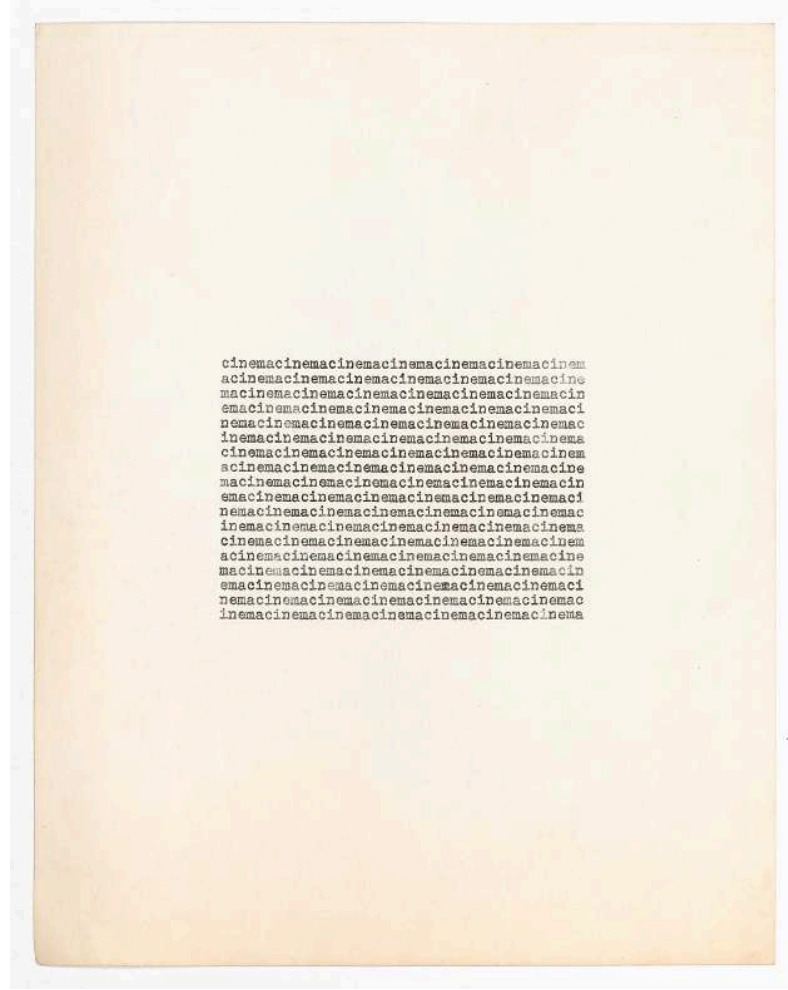

(C) Violette Garnier et Musashino Art University Museum \& Library, Japan. 
Figure 6 : Un des poèmes de Cimetière spatialiste envoyés par Pierre Garnier pour l'exposition ASA de 1973.

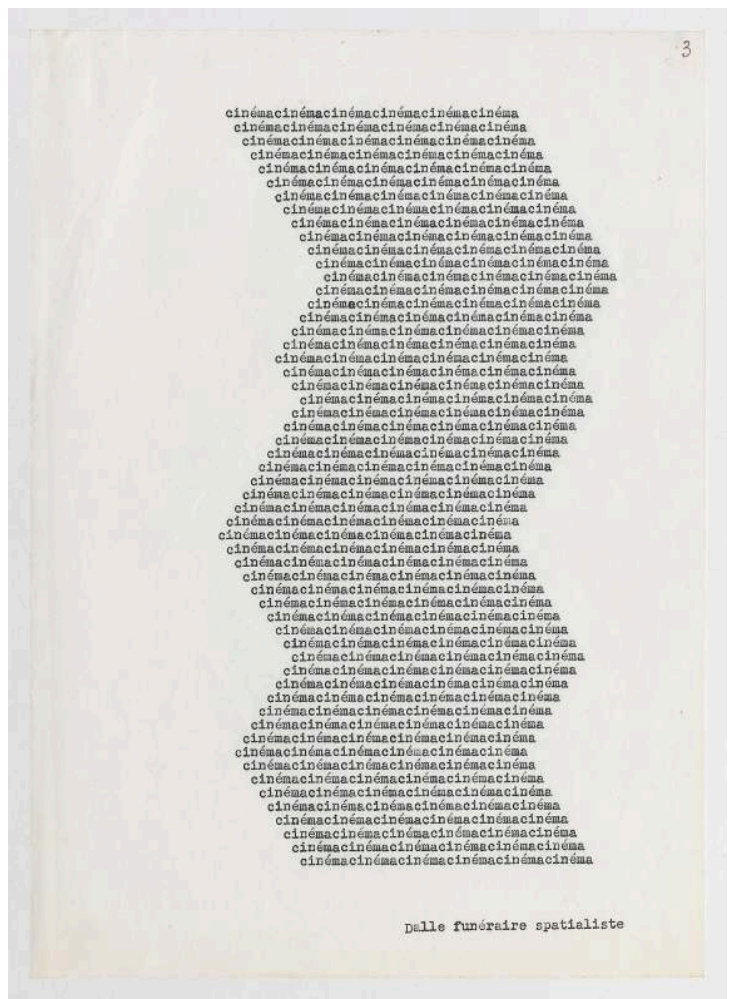

(c) Violette Garnier et Musashino Art University Museum \& Library, Japan.

Dans le sillage de Victor Vasarely, défenseur d'un «art fonctionnel » conçu pour être utilisable par tous et offert à la duplication, les poètes spatialistes conçoivent le poème comme un prototype, destiné à devenir un objet utile au terme d'une reproduction mécanique à grande échelle. "C'est un projet. Il est d'abord fabriqué à un seul exemplaire, puis peut être multiplié avec variation de formats mais conservation des rapports, souvent par application d'une instruction simple » (préface), expliquent-ils. Aucun architecte ne semble avoir tenté de remplir le programme de Prototypes. À quoi auraient pu ressembler une gare, un cimetière ou une tapisserie réalisés à partir de ces poèmes? À tout autre chose sans doute que les œuvres imprimées sur les feuilles, malgré la « conservation des rapports » d'un support à l'autre. Mais l'essentiel n'est pas là : l'intérêt de ces poèmes pour notre propos réside dans la décision des poètes de programmer leur création en vue d'une réalisation matérielle hors de la page, dans des espaces aux dimensions gigantesques. Conçu comme un prototype, le poème possède en germe les conditions de sa réalisation en dehors de son support et de son format d'origine.

\section{Le livre réinventé}

17 Une autre façon de sortir de la page est d'explorer l'espace du livre. La contribution d'Ilse et Pierre Garnier à l'art du livre est encore largement méconnue. Anne MœglinDelcroix affirme que les poètes concrets développèrent « un art de la page au détriment d'un art du livre» (80), en raison de leur méconnaissance des principes sur lesquels repose ce dernier : attachés à la tabularité de la page, à l'image plus qu'au discours, ils 
auraient ignoré la linéarité du livre et la dynamique qui lui est propre. Selon elle, « la plupart des livres de poésie concrète ne sont donc en réalité que des recueils de pièces poétiques " (83). Le spatialisme oblige à pousser la discussion un peu plus loin, et à nuancer pour le moins le propos.

Ilse et Pierre Garnier étaient en effet pleinement conscients de la spécificité du livre et des possibilités qu'il offre. «Ce qui se passe dans la suite des pages est aussi intéressant que ce qui se passe au sein de chacune », explique Pierre Garnier. Sans nullement se limiter aux rapports d'énergie entre les mots disposés sur la page, les poètes se sont progressivement « intéressés aux séries, aux formes qui évoluent de page en page, qui se meuvent dans l'espace de la page, aux glissements, introduisant un élément temporel, une évolution de la forme ou de la pensée » (Depétris, I. et P. Garnier 10). La linéarité du livre ne leur était pas étrangère, et ils s'employèrent au contraire à en tirer tout le profit possible. Pierre Garnier invite par exemple à « organiser le texte selon les pliures, les ruptures, les reliefs» («Position 4 du Spatialisme » 211) : le format pour lui est bien dispositif de création, un moyen d'inventer de nouvelles formes poétiques. Examinons par exemple quelques-unes des expériences menées par Ilse et Pierre Garnier pour faire du livre un objet en mouvement ${ }^{6}$.

\section{Délier}

19 Un livre se présente généralement sous la forme de feuilles assemblées, que le lecteur tourne les unes après les autres dans un ordre fixé d'avance. À l'exception notable de Poèmes mécaniques, les recueils de poèmes spatialistes publiés conjointement et sous leurs deux noms par Ilse et Pierre Garnier dans les années 1960 et 1970 sont dépourvus de reliure. Ils sont constitués de feuilles de papier alfa coloré, de format légèrement inférieur au A4, non numérotées, réunies dans une pochette. Il s'agit de Prototypes. Textes pour une architecture (1965), Othon III - Jeanne d'Arc. Structures historiques (1967), Esquisses palatines (1971) et Jardin japonais (1977). Le dispositif sera repris aussi pour le recueil Poèmes franco-japonais (1966) de Pierre Garnier et Seiichi Niikuni.

Certaines de ces œuvres sont fortement structurées. Dans Othon III - Jeanne d'Arc par exemple, la partie consacrée à Othon III suit un «plan » en trois parties (« Le message », «Portrait d'Othon III » et «Le Monde et l'action d'Othon III »), et les «planches » sont même numérotées dans la préface, alors que les feuilles proprement dites ne comportent pas de numéros (Fig. 7 et 8). 
Figure 7. Poème de Pierre Garnier. Ilse et Pierre Garnier. Othon III - Jeanne d'Arc. Structures historiques. Paris : André Silvaire, 1967.

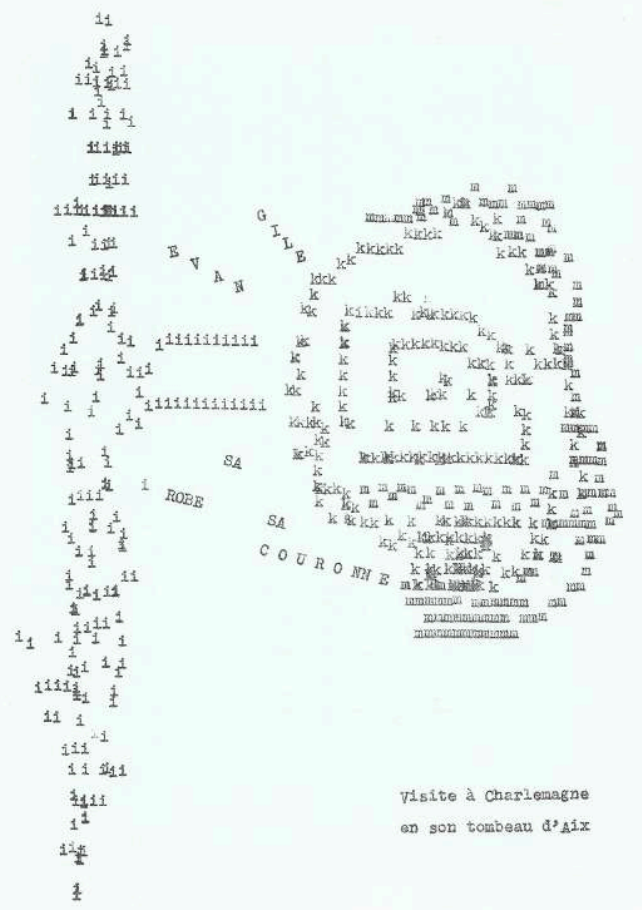

Figure 8. Poème d'Ilse Garnier. Ilse et Pierre Garnier. Othon III - Jeanne d'Arc. Structures historiques. Paris : André Silvaire, 1967.

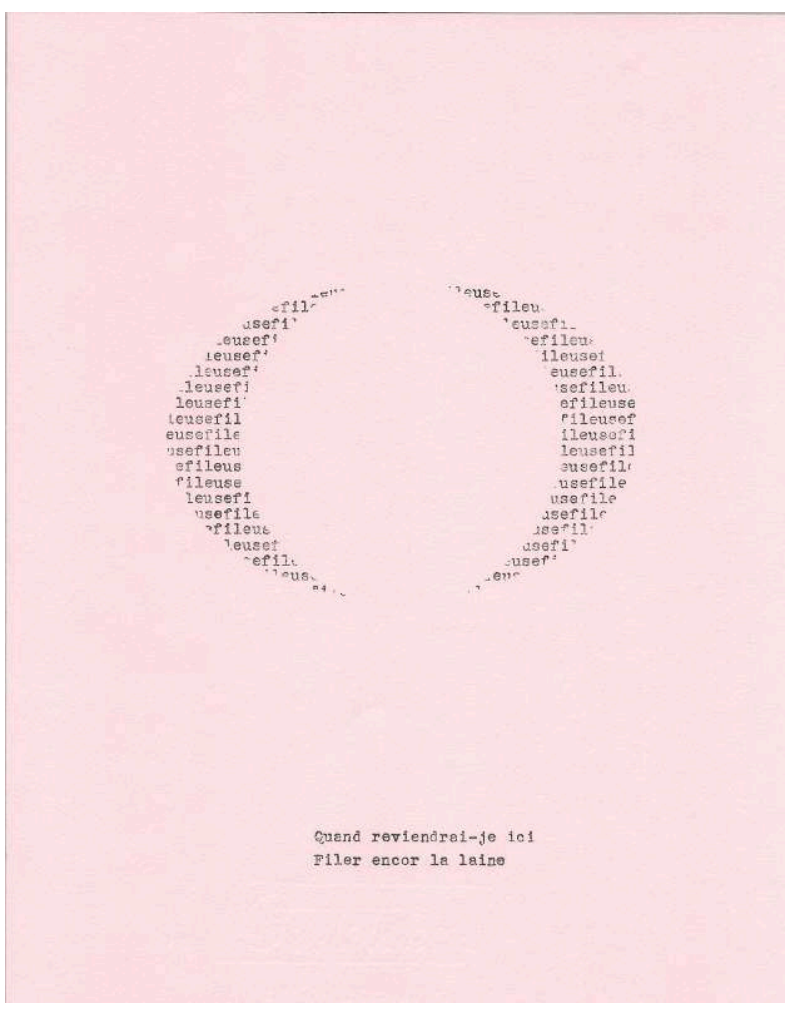


21 Dans Poèmes franco-japonais, l'ordre dans lequel sont classés les mots japonais dans le lexique peut être considéré comme un ordre possible des poèmes eux-mêmes dans le recueil. Reste que les poètes ont renoncé à attacher les feuilles ensemble, et on peut penser que ce choix, nullement contradictoire avec le soin apporté à la structuration interne des recueils, répond à plusieurs exigences. La souplesse et l'économie d'abord : l'absence d'assemblage permet d'imprimer les poèmes sur des papiers de couleurs différentes, d'ajouter ou d'enlever des feuilles au dernier moment. Au moins pour les recueils jusqu'à Esquisses palatines, ce choix correspond aussi au désir des poètes de produire une œuvre commune dans laquelle l'identité de chaque auteur resterait invisible au lecteur. Dans leur refus de tout narcissisme, les deux poètes qui composaient leurs œuvres séparément, les mélangeaient au moment de la publication. "C'était une œuvre à deux, les poèmes ne distinguaient pas l'auteur, du moins, le croyions-nous ", écrit Ilse Garnier (Jazz pour les yeux 39). Et puis, la mobilité des feuilles donne au lecteur la possibilité de les manipuler à sa guise, de les lire et de les regarder dans l'ordre qu'il souhaite. L'absence d'assemblage confère un cinétisme au moins virtuel au dispositif, et représente une des clés de la liberté du lecteur-spectateur.

\section{Déplier}

Le leporello constitue une modalité du livre plus cinétique encore que les feuilles séparées sous pochette : les feuilles collées les unes aux autres peuvent être tournées et former des doubles pages comme dans un livre standard, mais elles peuvent aussi être dépliées de manière différente pour faire apparaître l'équivalent de trois ou quatre pages, voire la totalité du livre. Pierre et Ilse Garnier y eurent chacun recours dans des œuvres évoquant elles-mêmes un mouvement, en l'occurrence un déplacement dans un paysage : Pierre Garnier dans Congo - Poème pygmée, poème spatial en 1980, et Ilse Garnier dans Ermenonville, partition pour un promeneur solitaire (création d'un paysage sonore) en 1984.

Les deux œuvres sont matériellement très proches : Congo est fait de 6 longues feuilles de papier collées et pliées de manière à constituer l'équivalent de 40 feuilles de format A4 verticales, imprimées d'un seul côté; Ermenonville, de 6 longues feuilles pour l'équivalent de 18 feuilles de format A4 horizontales. Le format du leporello permet la superposition simultanée de trois mouvements : celui du corps qui se promène au bord de l'eau, celui de la main qui tourne les pages, et celui du regard qui parcourt le poème de feuille en feuille. La page n'est plus l'unité du poème, pas plus que la double page : le sens de l'œuvre est indissociable de la continuité de son support, continuité d'ailleurs variable selon la manière dont le lecteur-spectateur tourne et déplie les pages, tant il est vrai que, comme le rappelle Isabelle Chol, «manipuler autrement le livre suppose aussi de manipuler autrement le texte, à partir de parcours de lecture multiples » (25). Le regard est appelé à suivre les lignes, les flèches, les enchaînements de mots d'une page à l'autre. Les deux livres se caractérisent aussi par le rapport que le dispositif entretient avec la thématique abordée. Inspiré entre autres par une promenade à Hanovre ainsi que par le désir « d'isoler le courant de la pensée - et de faire de son courant un poème spatial » (épigraphe), Congo - Poème pygmée, poème spatial évoque le cours du fleuve : sa longueur est rendue par le continuum du support, l'écoulement de l'eau par des flèches tracées à certains endroits de la composition, les berges par deux traits horizontaux continus en haut et en bas (Fig. 9). 
Figure 9 . Pierre Garnier. Congo - Poème pygmée, poème spatial. Paris : André Silvaire, 1980.

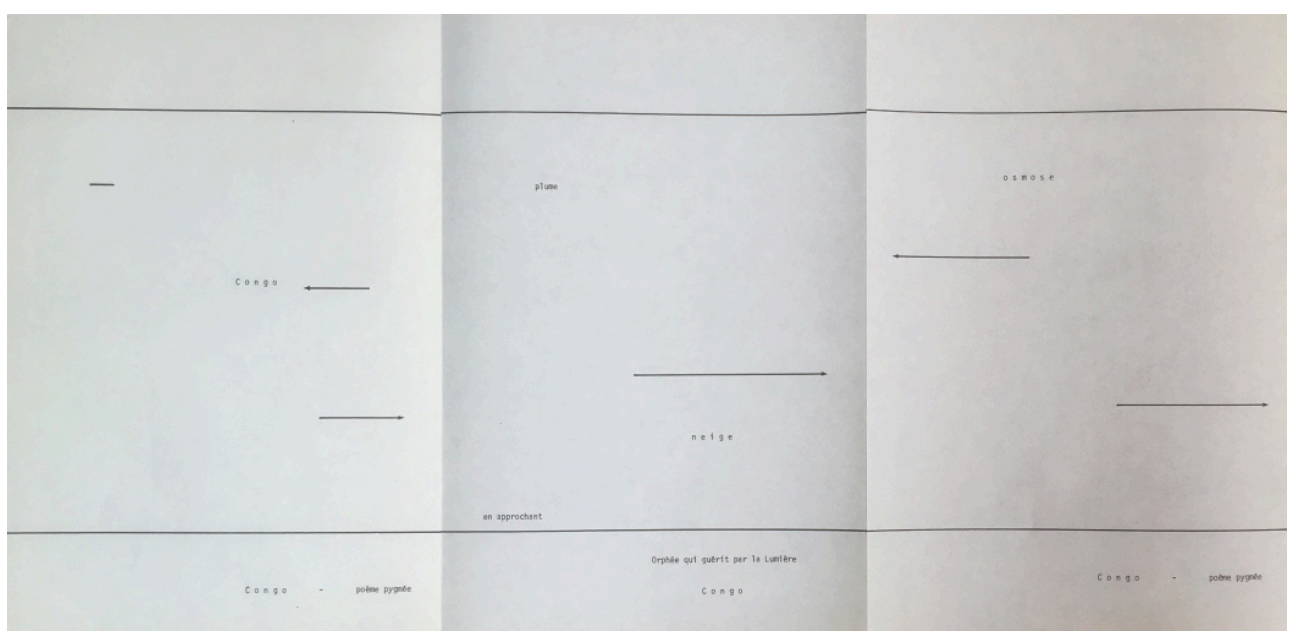

Pensé avec Jean-Jacques Rousseau, qui termina sa vie à Ermenonville et y fut inhumé, Ermenonville, partition pour un promeneur solitaire (création d'un paysage sonore) offre au lecteur une promenade visuelle, comparable à celle d'un marcheur qui découvrirait le paysage. La ligne médiane horizontale qui traverse tout le leporello peut faire penser à la fois à une eau courante et à une portée musicale, d'autant qu'elle est parfois accompagnée d'autres segments eux aussi horizontaux, et que le vocabulaire du son est omniprésent dans l'œuvre. Certains éléments plastiques ou graphiques commencés sur une page se poursuivent sur la suivante (Fig. 10).

Figure 10. Ilse Garnier. Ermenonville, partition pour un promeneur solitaire (création d'un paysage d'écriture sonore). Paris : André Silvaire, 1984.

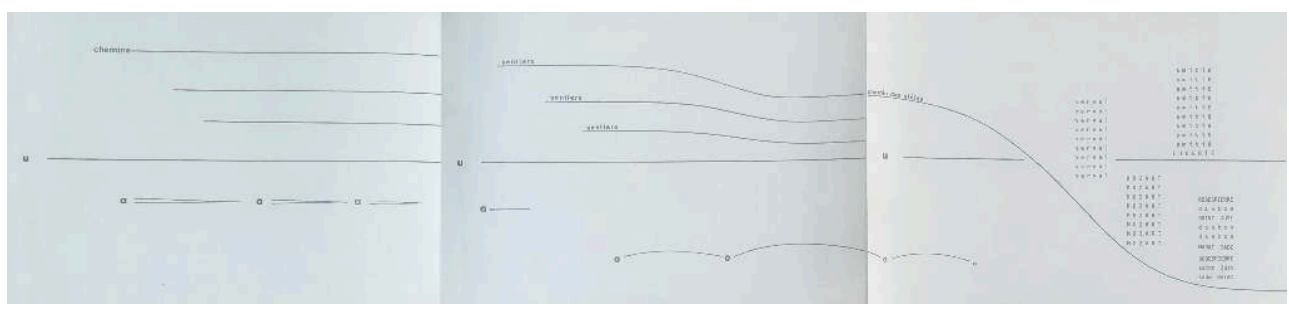

La manière dont Congo et Ermenonville furent présentés dans des expositions, en 1980 et 2002 respectivement, souligne l'importance qu'y occupe la linéarité. Avant de devenir un leporello, Congo fut montré au C.R.D.P. de Rouen « dans une rotonde conçue pour lui : le visiteur assis au centre pouvait suivre le courant de la (de sa) pensée » (préface n.p.), tandis qu'Ermenonville fut déplié et collé sur une ellipse métallique suspendue au plafond, autour de laquelle le lecteur/spectateur tournait (Fig. 11). Il ne s'agissait pas alors de tourner les pages du livre, mais le mouvement n'était pas pour autant absent du processus. Il s'était simplement déplacé, au centre ou à l'extérieur de l'œuvre. 
Figure 11. Ilse Garnier. Ermenonville, partition pour un promeneur solitaire (création d'un paysage d'écriture sonore). Paris : André Silvaire, 1984.

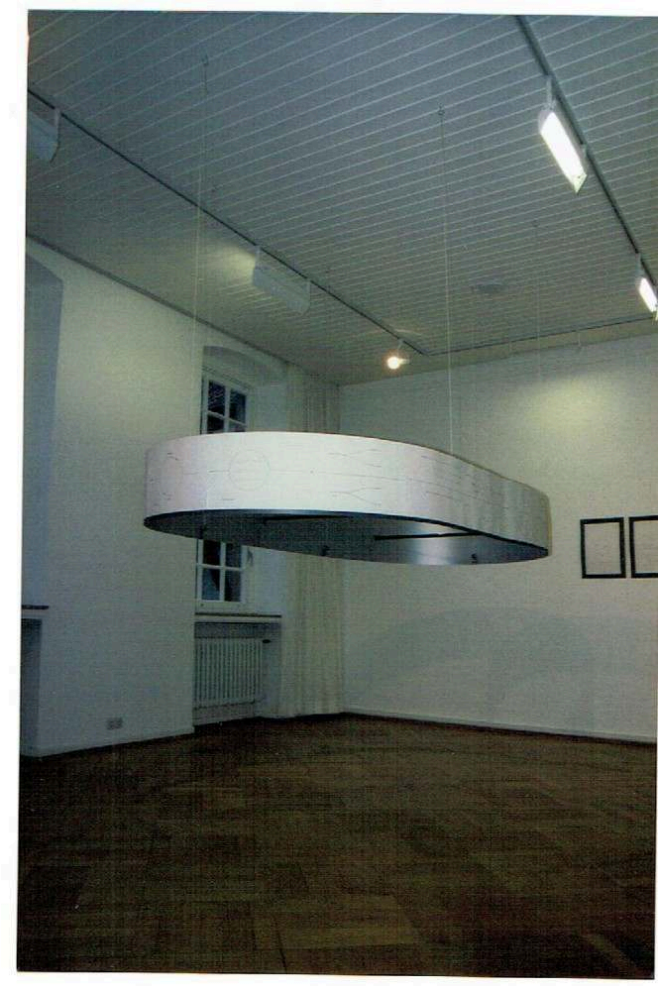

Photo prise lors de l'exposition ilse garnier rythmes et silence, organisée par le Bielefelder Kunstverein (Allemagne) du 4 mai au 16 juin 2002. Archives familiales Ilse et Pierre Garnier.

(C) Violette Garnier.

\section{Feuilleter}

Partageant avec le leporello l'idée de continuum, le folioscope (flip book) est un petit livret d'images où les gestes d'un sujet sont décomposés chronologiquement, et qui, feuilleté rapidement, procure à l'œil l'illusion du mouvement. Ilse Garnier possédait un petit livre de ce genre, dans lequel une suite de photos permet de voir les mouvements d'une danseuse. Le procédé est ancien puisqu'il remonte au XIX ${ }^{e}$ siècle, mais Pierre et Ilse Garnier en tirent des effets surprenants dans Tristan et Iseult (poème spatial) et dans La Meuse.

27 Tristan et Iseult (1981) se présente sous la forme d'un volume de feuilles A4 imprimées au recto (un poème par page), non numérotées, et reliées à la colle sous une couverture en papier fort (Fig. 12). La thématique amoureuse, fréquente dans la poésie de Pierre Garnier, s'inscrit ici dans un dispositif pour le moins inattendu. 
Figure 12. Pierre Garnier. Tristan et Iseult, Poème Spatial. Paris : André Silvaire, 1981.

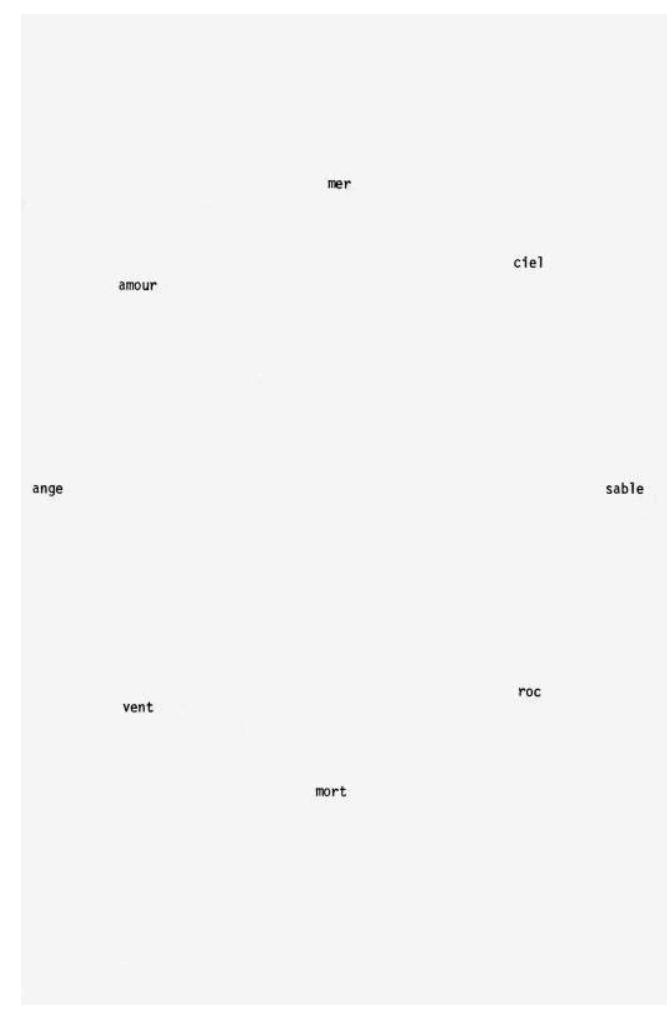

Dans une lettre au réalisateur et photographe Christian Janicot, le poète affirme en effet qu'il fut "pensé pour une vidéo » (Simon-Oikawa 2019b, 8). Dans sa préface, Ilse Garnier détaille le mode d'emploi du livre :

Les progressions de mots doivent être vues d'un coup - il faut poser les feuilles les unes à côté des autres, de façon que la suite se découvre lentement ou bien alors produire en feuilletant rapidement un développement cinétique. Ce sont la plupart $\mathrm{du}$ temps des progressions ouvertes qui se poursuivent dans l'espace au-delà $d u$ bord de la page - progressions cosmiques dans un espace spirituel.

Poser les feuilles les unes à côté des autres est impossible au lecteur qui n'a accès qu'à l'œuvre imprimée, puisque celle-ci est reliée. Mais il peut tourner les pages rapidement. Les poèmes, disposés dans le livre sous forme de séries, se transforment alors peu à peu sous ses yeux. La modification est particulièrement remarquable dans le cas d'une série de neuf poèmes disposés en forme de roue. Dans le premier poème on lit, en partant du haut et dans l'ordre des aiguilles d'une montre, un ensemble de mots qui se rattachent à la matière de Bretagne : " mer ", « ciel ", « sable ", « roc ", « mort ", « vent ", « ange ", " amour ». Les huit autres suivent, orientés différemment : le mot situé au point le plus haut est remplacé à la page suivante par celui qui se trouvait directement à sa gauche, jusqu'au retour au point de départ. Vue à travers le défilement rapide des pages, la roue semble tourner véritablement (Simon-Oikawa 2019b, 56-63).

Dans La Meuse, petit livre $(24,5 \times 13 \mathrm{~cm})$ dont le format paysage peut être mis en relation avec le cours du fleuve et la demi-teinte gris bleuté avec la couleur de l'eau, Ilse Garnier explore le thème aquatique déjà abordé dans Ermenonville, mais en s'inspirant d'une œuvre de Ian Hamilton Finlay. Dans le cadre d'une manifestation organisée du 11 au 15 septembre 1986 pour la $\mathrm{XV}^{\mathrm{e}}$ Biennale de poésie de Liège, Finlay avait en effet imaginé de faire flotter sur des canaux des feuilles de papier «portant chacune un mot, une expression, pour 'poétiser' le mouvement des eaux », et Ilse 
Garnier avait gardé en mémoire cet «événement poétique : ce jeu entre l'eau, les feuilles et les mots du poète emportés au gré du courant " (Jazz pour les yeux 310). La Meuse fait alterner des pages sur lesquelles les verbes «flotter» et "couler " sont disposés horizontalement à des hauteurs variées sur la page (Fig. 13); d'autres sur lesquelles le mot « eaux » est associé à des adjectifs comme « lisses », "profondes » ou « immobiles»; d'autres encore qui contiennent des expressions écrites au letraset ou structurées autour de blocs.

Figure 13. Ilse Garnier. La Meuse. Paris : André Silvaire, 1991.

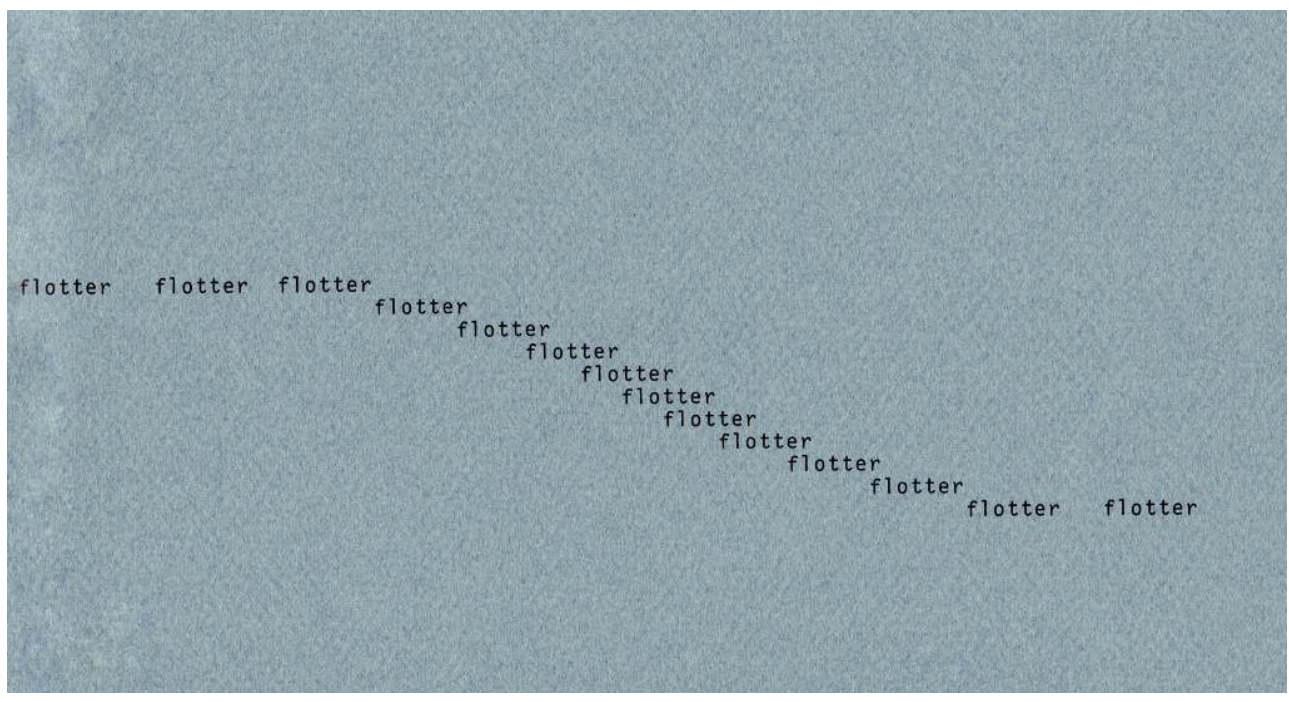

La composition de l'œuvre suit le principe de la variation musicale, mais c'est au folioscope qu'Ilse Garnier fait référence pour expliquer le fonctionnement de son livre, lorsqu'elle indique dans un entretien: "Le petit livret forme un ensemble qu'on appelait cinéma et que l'on passe en vitesse en regardant très vite ${ }^{7}$ ». En feuilletant les pages, le lecteur-spectateur voit le fleuve se mouvoir sous ses yeux. On est frappé par la simplicité des procédés mis en œuvre. Le cinétisme des poèmes ne repose sur aucun appareillage particulier : Ilse et Pierre Garnier se sont attachés à travailler le livre dans ce qu'il a de plus banal et immédiat, des feuilles qui se tournent.

\section{D'un format, l'autre}

Les poètes produisirent tous les deux des livres d'artiste, mais c'est sans conteste Ilse Garnier qui fut la plus active dans ce domaine. Elle réalisa des œuvres de différents formats, depuis le raisin dans Fensterbilder. Ein Stundenbuch (Royère), jusqu'à des carrés plus petits dans Winterlandschaft mit Vögeln $(25 \times 25 \mathrm{~cm})$ (Simon-Oikawa 2019a). Elle créa aussi pour des expositions des poèmes aux dimensions parfois importantes, comme les 17 poèmes de Quartett - ein Zahlentext qui, dans leur version la plus grande, mesurent 50 $\mathrm{cm}$ de côté (il existe aussi une version plus petite, de $25 \mathrm{~cm}$ de côté) et combinés quatre à quatre forment des damiers en noir et blanc d'1 $\mathrm{m}$ de côté. Ilse Garnier travailla les formats avec un soin tout particulier selon des modalités multiples, notamment la récriture de poèmes dans des formats différents, l'introduction de la tridimensionnalité dans des supports bidimensionnels, et la migration de poèmes sur des supports multiples. 


\section{Récritures} permet des comparaisons intéressantes. C'est le cas d'Éros et Psyché, ensemble consacré à l'histoire de ces deux divinités, et dans lequel chaque poème, composé d'éléments plastiques ou textuels en français, est accompagné d'une phrase, elle-même écrite en français ou en allemand, la langue maternelle d'Ilse Garnier ${ }^{8}$. On connaît de cette œuvre quatre versions élaborées en 1989 : l'une que nous appellerons A, en français, sur papier fort $(65 \times 50 \mathrm{~cm})$; une version $B$, en allemand, sur un mélange de papiers artisanaux découpés à la main ( $38 \times 23 \mathrm{~cm}$ environ, faibles variations d'une feuille à l'autre); une version $\mathrm{C}$, identique à la version $\mathrm{B}$, mais en français (trois poèmes reproduits dans Gappmay 2004, 308-10); et une version D, en français, connue par des photocopies, les originaux ayant disparu (format A4). Dans chacune des quatre versions, les poèmes renvoient aux mêmes scènes. Ils sont au nombre de quatorze, sauf dans la version $\mathrm{D}$ où manque «Éros poursuit sa proie ». Il n'est pas impossible que d'autres versions existent par ailleurs. On sait qu'une version française fut exposée à la Galerie J. J. Donguy du 13 février au 17 mars 1990, qu'une version en allemand fut montrée à Gmunden (Autriche) et que la version D fut utilisée pour l'anthologie Jazz pour les yeux, livre au format carré $(21 \mathrm{x} 21 \mathrm{~cm})$. Ilse Garnier réalisa nombre de ses œuvres à l'occasion de manifestations poétiques ou d'expositions, et les versions grand format ont peut-être été exécutées précisément dans ce contexte.

En labsence de documentation précise sur la genèse de ces quatre versions, il est difficile d'établir leur chronologie exacte. Leur comparaison est néanmoins instructive, et éclaire notamment le traitement différencié qui y est fait du blanc. Dans les versions A, B et C, les espaces laissés libres dans la composition occupent une place majeure. Dans la version A du poème "La colombe sacrifiée », la figure constituée d'un cercle rouge entourant les mots "plumes », «ailes », "flamme » et "cendres », et la phrase qui l'accompagne, sont placées sur la partie droite de la feuille, très espacées l'une de l'autre: la partie gauche et le centre de la feuille sont laissés vierges, tout comme l'espace à droite qui sépare la figure et la phrase (Fig. 14). 
Figure 14. Ilse Garnier. Éros et Psyché. 1989 (version A). Archives familiales Ilse et Pierre Garnier.

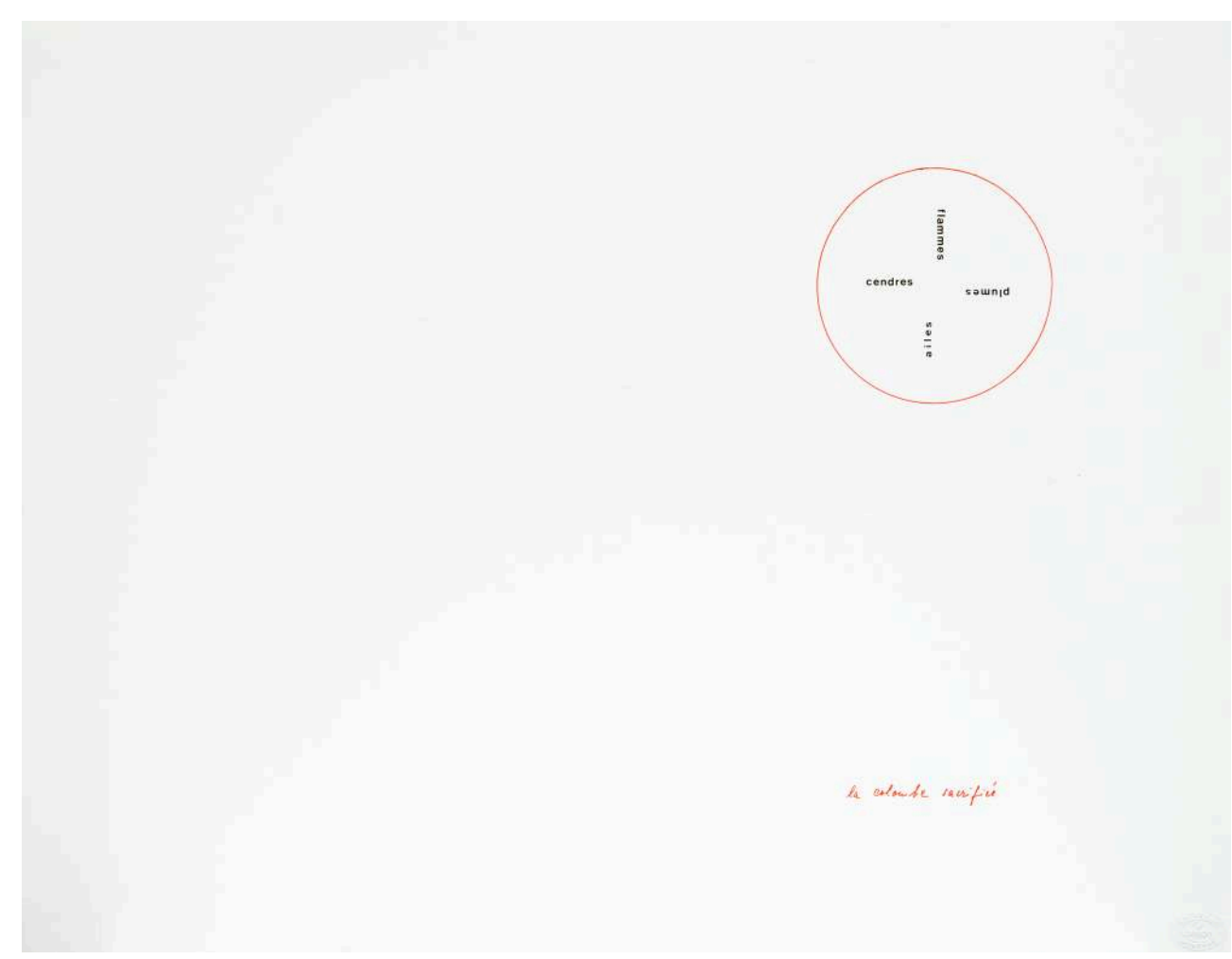

(C) Violette Garnier.

Dans la version B (« die gespferte Taube »), les mots entourés par le cercle sont mis en page de manière plus dynamique, le cercle et la phrase sont placés en deux coins opposés de la feuille disposée horizontalement, mais le blanc reste majeur : il occupe tout l'espace compris entre le coin supérieur gauche et le coin inférieur droit (Fig. 15).

Figure 15. Ilse Garnier. Eros und Psyché. 1989 (version B). Archives familiales Ilse et Pierre Garnier.

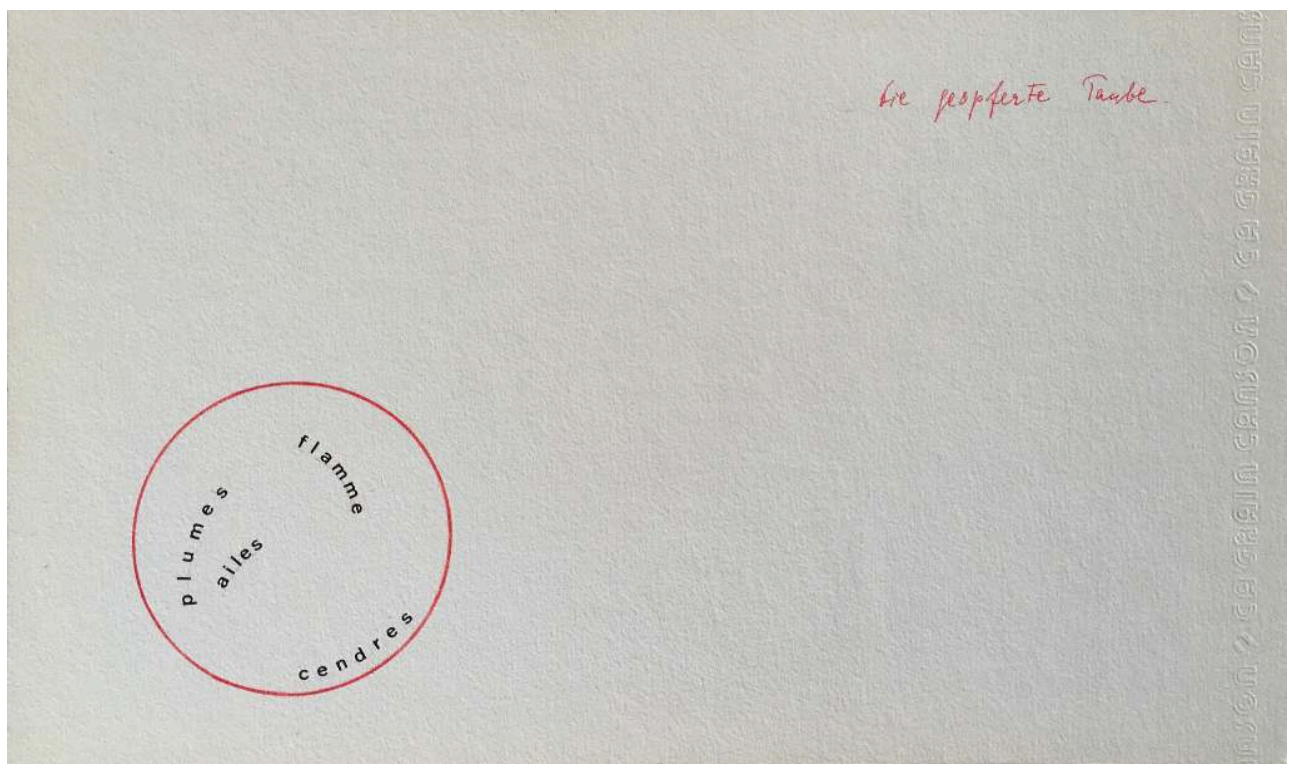

(C) Violette Garnier 
Dans la version A4, Ilse Garnier a ajouté une flèche qui rappelle l'arc et les flèches dont est muni Éros, mais surtout, pour faire entrer le poème dans un format plus réduit que l'espace d'origine, elle a été conduite à rapprocher considérablement les éléments pastiques et textuels (Fig. 16). Le blanc y devient secondaire.

Figure 16. Ilse Garnier. Éros et Psyché. 1989 (version D). Reproduit dans Jazz pour les yeux (n.p.).

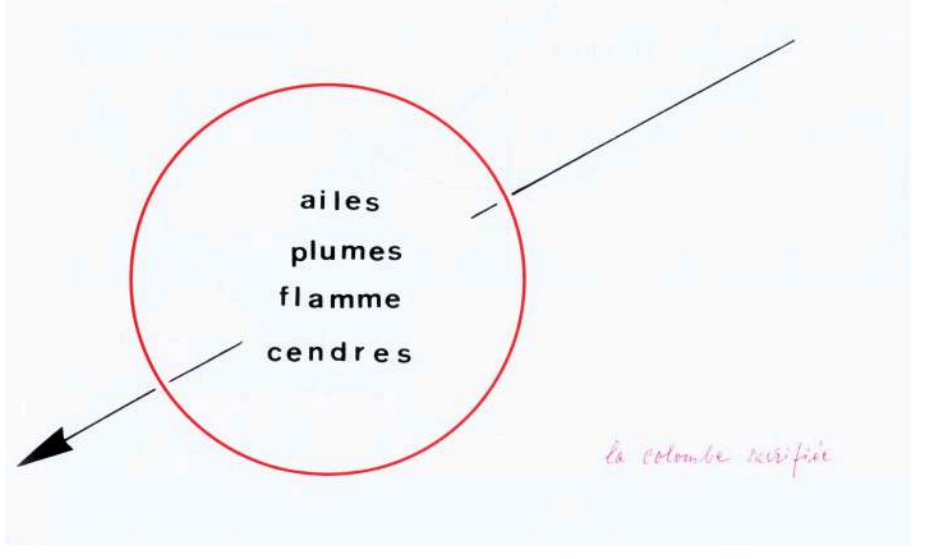

\section{Du bidimensionnel au tridimensionnel}

Quand un support bidimensionnel se transforme en un objet tridimensionnel, la perception qu'en a le lecteur est elle aussi profondément modifiée. On connait d'Ilse Garnier cinq cubes poétiques en papier coloré sous plexiglas, un pour chacune des voyelles $\mathrm{a}, \mathrm{e}, \mathrm{i}, \mathrm{o}, \mathrm{u}$. Mais en matière de tridimensionnalité Puzzle-Alphabet est sans doute sa création la plus surprenante et la plus ambitieuse. L'œuvre se présente sous la forme de feuilles de papier fort au format A4, une pour chaque lettre de l'alphabet, destinées à former un jeu de cubes. Chaque feuille entièrement réalisée à la main contient le patron d'un cube, dont l'une des faces porte la lettre de l'alphabet qui figure dans les mots français, anglais et allemands présents sur les autres, et qui est laissée en blanc dans l'alphabet inscrit sur le carré qui lui fait face. Sur le carré central sont collés un ou deux autres carrés de papier Canson, plus petits et de couleur. À l'intérieur de ce schéma, une grande variété poétique et graphique se donne à voir, chaque carré constituant en soi un petit poème visuel qui souvent joue sur un effet de mimétisme. Pour la lettre $O$ par exemple, Ilse Garnier a disposé le mot « œil » de manière à suggérer la forme d'un œil, introduit une courbe dans le mot allemand «mond» (lune) pour évoquer un croissant de lune, et mis les deux « 0 » du mot «look » entre parenthèses pour donner à voir deux yeux (Fig. 17). Chaque feuille devait être découpée et collée 
sur un cube de bois de $8 \mathrm{~cm}$ de côté, et une boîte pour ranger les cubes est elle aussi conservée (Fig. 18).

Figure 17. Ilse Garnier. Puzzle-Alphabet, Jeu de Cubes, 1988. Archives familiales Ilse et Pierre Garnier.

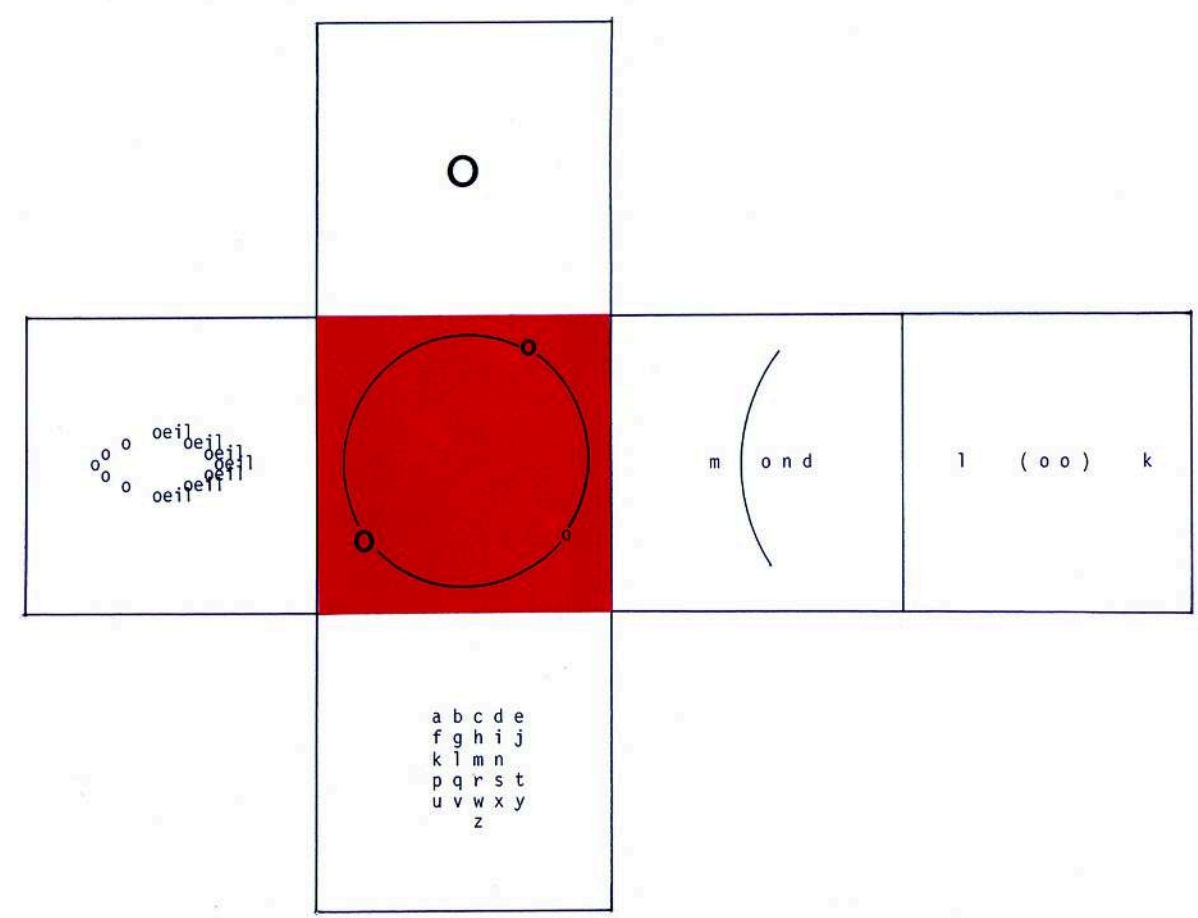

(C) Violette Garnier et Thierry Chauveau. 
Figure 18. Ilse Garnier. Puzzle-Alphabet, Jeu de Cubes, 1988.

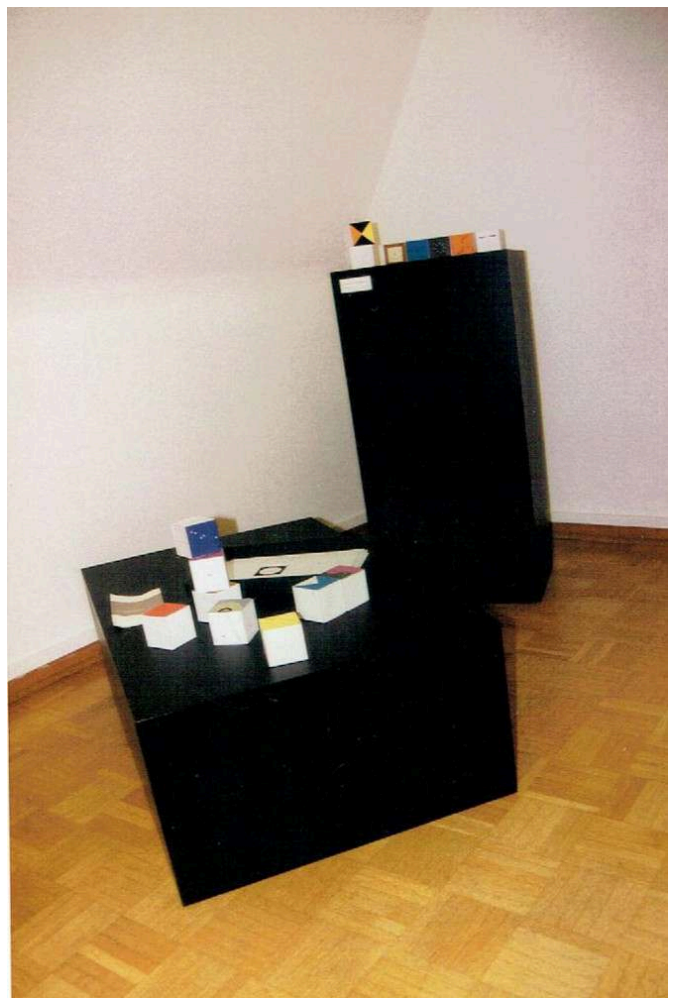

Photo prise lors de l'exposition ilse garnier rythmes et silence, organisée par le Bielefelder Kunstverein (Allemagne) du 4 mai au 16 juin 2002. Archives familiales Ilse et Pierre Garnier.

(C) Violette Garnier.

Dira-t-on que seul compte le produit final, et que le destin de l'objet en deux dimensions se résume à disparaître au cours du processus de montage ? La structure de chaque feuille est au contraire particulièrement intéressante ici. Les patrons géométriques ne sont pas sans rappeler en effet un autre objet associé lui aussi à la culture enfantine, la marelle, dont le modèle le plus fréquent dit "cathédrale ", consiste en deux bandes croisées constituées de carrés, et de longueur inégale. La comparaison se justifie d'autant plus que, un an avant Puzzle-Alphabet, lors du festival Europalia Autriche organisé en Belgique en octobre 1987, Ilse Garnier avait proposé une œuvre éphémère sous la forme de cinq marelles poétiques tracées sur le sol et destinées à s'effacer peu à peu sous les pas des visiteurs (Édeline 2018). Consacrées chacune à une des voyelles $\mathrm{a}, \mathrm{e}, \mathrm{i}, \mathrm{o}, \mathrm{u}$, elles rappelaient, et c'était même là leur première raison d'être, la devise AEIOU imaginée par l'empereur Frédéric III et dont l'une des interprétations possibles est Austria Est Imperare Orbi et Universo (Le destin de l'Autriche est de commander au monde et à l'univers). Mais elles signalaient aussi l'attachement d'Ilse Garnier à la thématique cosmique : le jeu de marelle ne consiste-t-il pas à aller de la Terre au ciel ? Parallèlement à ces marelles au sol, Ilse Garnier avait d'ailleurs préparé une série de 27 diapositives poétiques intitulées «marelles du ciel», qui étaient projetées dans l'exposition et prenaient pour thème les lettres $a, e, i, o, u$ (Édeline 2019).

Proximité formelle (combinaison de bandes croisées), thématique (lettres de l'alphabet), métaphysique (voyage dans l'espace et cheminement spirituel pour les marelles, figuration de la structure de l'univers pour le patron géométrique), 
chronologique (1987 pour le festival Europalia Autriche, 1988 pour Puzzle-Alphabet) et même géographique puisque Puzzle-Alphabet fut publié par un éditeur belge, PuzzleAlphabet et les marelles renvoient aussi au rêve qu'Ilse Garnier nourrissait quand elle était enfant de devenir architecte. La précision et la minutie de ses constructions témoignent de son sens de la matière et de l'espace, ou plutôt des espaces. Ils disent son désir de construire un monde de signes en poésie à l'aide d'un format saisi non pas seulement comme forme mais comme condition de possibilité d'un usage spécifique de cette forme, en l'occurrence ici une manipulation conforme à des règles: pliage géométrique dans un cas, avancée selon un lancer de caillou dans l'autre.

\section{De la feuille à l'écran}

Des marelles au puzzle, Ilse Garnier approfondit une inspiration sans qu'il soit possible de parler de citation ou même de reprise. Il arrive parfois que les métamorphoses d'une œuvre de départ soient plus explicites. Prenons Blason du corps féminin, le premier recueil de poèmes qu'Ilse Garnier publia sous son seul nom en 1979. Inspiré à la fois par un genre littéraire en vogue à la Renaissance, la description des parties du corps féminin, et par un voyage qu'Ilse Garnier fit au Sénégal où elle eut l'occasion d'observer les femmes, « leurs gestes, leur aisance, leur élégance » (I. Garnier, chiendents 15), Blason du corps féminin est construit tout entier autour du mot « corps » : " corps ailé », " corps lune ", « corps fécondé ", " corps mutilé ", toute la vie des femmes est évoquée, de manière littérale ou figurée (Fig. 19). Constituée de 47 feuilles non reliées sous pochette, la série est aussi et de manière plus radicale encore une longue variation sur le « $\mathrm{O}$ », traité à la fois comme lettre alphabétique et comme forme géométrique.

Figure 19. Ilse Garnier. Blason du corps féminin. Paris : André Silvaire, 1979.

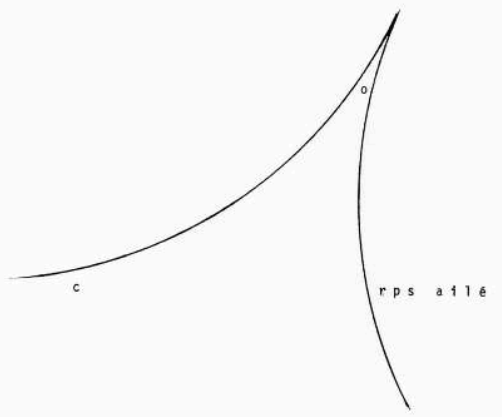


Le recueil connaît une première métamorphose sept ans plus tard, en 1986, lors de la $\mathrm{xV}^{\mathrm{e}}$ Biennale de poésie de Liège déjà évoquée plus haut. Ilse Garnier présente en effet à cette occasion une séquence de 85 diapositives intitulée Voyage cosmique, qui donnent à voir des poèmes visuels de sa création, dont plusieurs sont tirés de Blason du corps féminin. La série, montrée sous une tente à l'aide de deux projecteurs faisant défiler les diapositives sur un écran courbe et à des rythmes différents, évoque un voyage de la Terre vers l'univers. De la feuille de papier à la diapositive, les poèmes ont changé de support et de dimensions (les cadres mesurent $5 \times 5 \mathrm{~cm}$ ). Ils ont aussi changé de couleur : les poèmes originaux en noir sur fond blanc sont transposés sur la pellicule en blanc sur fond noir. Selon la distance à laquelle le poème a été photographié, selon aussi que la photo a été prise verticalement ou horizontalement, l'image projetée donne à voir des poèmes en entier ou d'autres tronqués. Certains poèmes apparaissent aussi plusieurs fois sous des formes différentes, sans compter que l'ordre dans lequel les poèmes sont montrés est contraint, alors qu'il restait flexible dans le cas du recueil puisque les feuilles ne sont pas reliées (Fig. 20 et 21). À la faveur d'un changement de medium, c'est donc une transformation généralisée de la lecture et de la contemplation des poèmes qui s'est opérée.

Figure 20. Ilse Garnier. Tirage photographique de la dispositive $\mathrm{n}^{\circ} 30$. Archives familiales llse et Pierre Garnier.

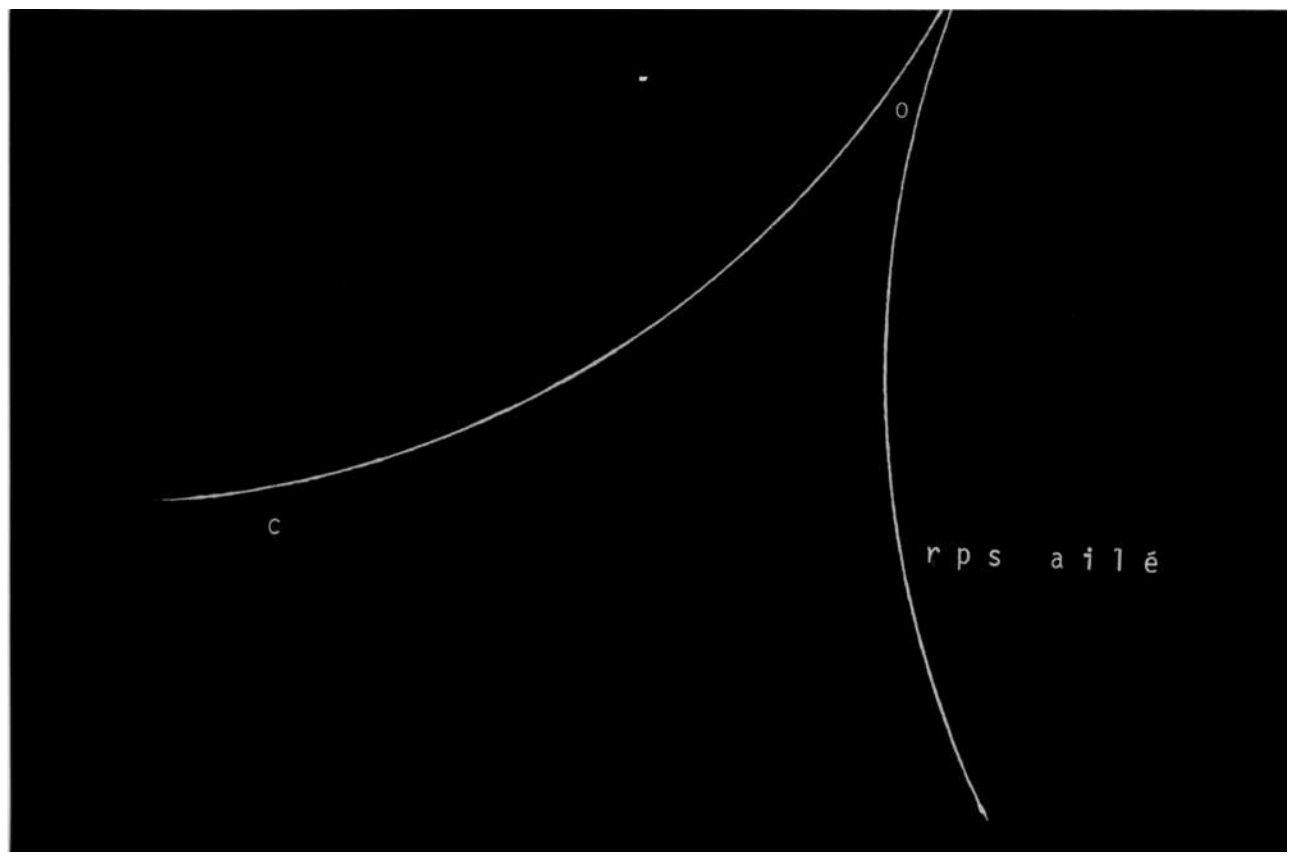

(c) Violette Garnier. 
Figure 21. Ilse Garnier. Tirage photographique de la dispositive $n^{\circ} \mathbf{4 5}$. Archives familiales llse et Pierre Garnier.

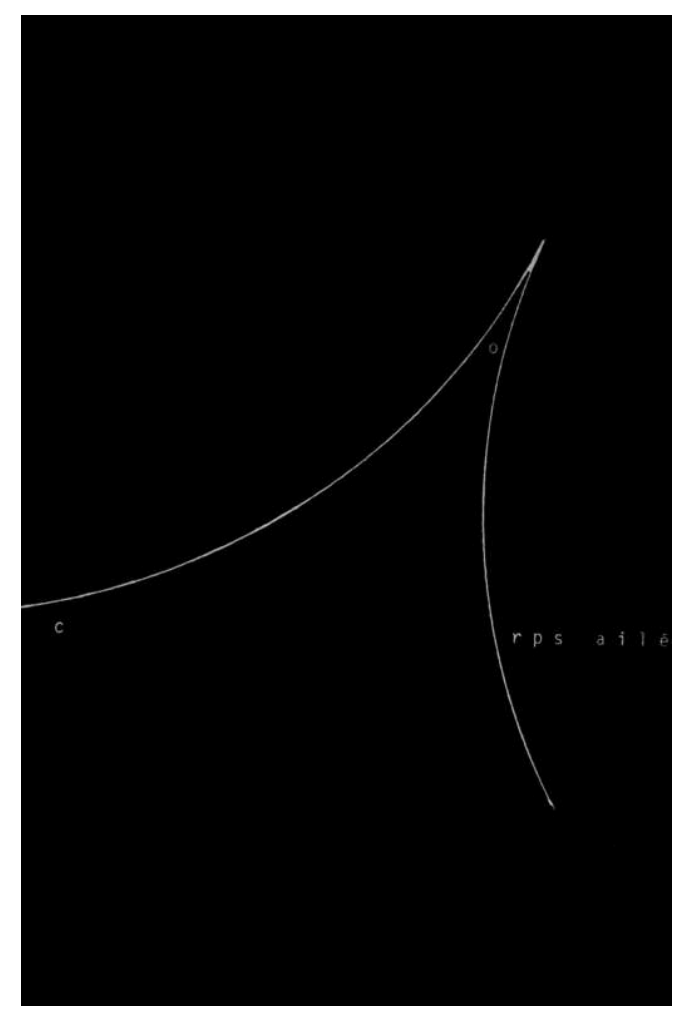

(c) Violette Garnier.

Un nouveau tournant a lieu en 1993, avec un scénario de «poème visuel cosmique » intitulé Poème cinématographique, qui reprend plusieurs poèmes, photocopiés et collés dans un texte à l'intention d'un éventuel réalisateur (V. Garnier), et surtout en 1996, quand Ilse Garnier réalise deux " ciné-poèmes », eux aussi sur le thème du voyage de la terre vers l'univers. Ces deux ciné-poèmes se présentent sous la forme de feuilles de papier épais type Canson de couleur ivoire, découpées au format A4, et orientées horizontalement au format paysage, conservées sous pochettes plastique dans deux classeurs. Chaque feuille contient des poèmes réalisés au letraset noir, à l'encre de Chine et à la mine de graphite, collés sur du papier photocopie blanc ou sur du Canson bleu (deux teintes), des éléments graphiques réalisés avec une grande minutie, des photographies, et un commentaire dactylographié qui indique comment les poèmes devraient être filmés et projetés. Les photographies collées sur les pages $(9 \times 13 \mathrm{~cm})$ sont toutes des tirages réalisés à partir de diapositives du Voyage cosmique de 1986 (Fig. 22) ${ }^{9}$. "Le moyen technique employé crée la poésie autant que le poète", affirmait Pierre Garnier dès 1962 (P. Garnier, « Manifeste pour une poésie nouvelle, visuelle et phonique » 6). Les créations multimédiales d'Ilse Garnier le montrent bien. De Blason du corps féminin aux "ciné-poèmes ", la reprise des poèmes va de pair avec l'exploration de nouveaux supports, sans qu'il soit possible de dire si la volonté de reprendre les poèmes dans un autre cadre a rendu nécessaire la recherche de nouveaux supports, ou si c'est le désir d'investir de nouveaux supports qui a conduit à la métamorphose des poèmes, les deux mouvements se produisant sans doute simultanément. 
Figure 22. Ilse Garnier. Ciné-poème. 1996. Archives familiales Ilse et Pierre Garnier.

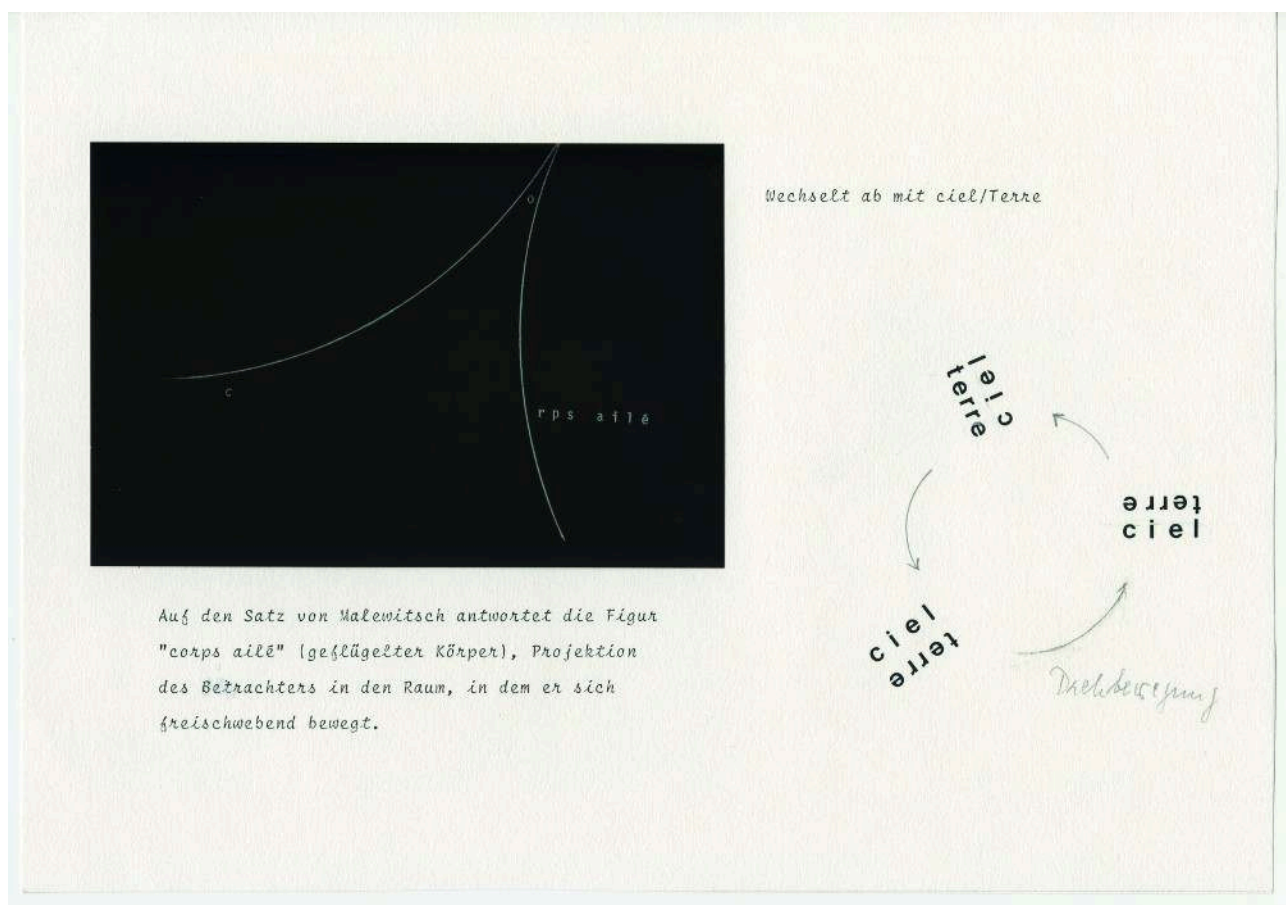

(c) Violette Garnier

Au terme de ce parcours, on espère avoir montré que la production visuelle des poètes spatialistes ne se limite pas à des suites de poèmes présentés sur des pages séparées, comme l'affirme Anne Mœglin-Delcroix à propos de la poésie concrète. Bien que marquée par les arts plastiques, l'esthétique d'Ilse et Pierre Garnier n'est pas tout à fait celle du tableau. Leur intérêt pour le mouvement, leur recherche de supports adaptés au monde moderne, mais aussi leur souci de produire des œuvres accessibles au plus grand nombre, les ont conduits à explorer les possibilités de la page et du livre en faisant preuve à chaque fois d'une économie de moyens remarquable. À la fois contrainte et condition de la création, le format fait l'objet chez eux d'une attention particulière, justifiée par une esthétique de l'espace compris comme élément constitutif $\mathrm{du}$ poème. Leur recherche de formats appropriés aux principes du spatialisme et leur exigence de simplicité les ont souvent conduits à privilégier des supports facilement accessibles, revisités toutefois par des pratiques que l'on n'hésitera pas à qualifier de transgressives. Elle les a parfois poussés, et ce point est particulièrement marqué chez Ilse Garnier, à reprendre un même poème sur différents supports, à expérimenter les possibilités offertes par le papier et par la diversité des moyens à leur disposition, de la page à l'écran de projection: l'espace chez elle est polymorphe, pluridimensionnel, profondément poétique. L'espace spatialiste ne renvoie pas seulement à un thème ou à un support : il est une préoccupation constante, à toutes les étapes de la construction et de la réception du poème. Les jeux de format en apportent la preuve : par eux les constellations du ciel étoilé s'incarnent en poèmes. 


\section{Ouvrages cités}

BUSCHINGER, Philippe. «La poésie spatialiste ou l'œuvre au blanc ». Pierre et Ilse Garnier : la poésie au carrefour des langues. Dir. Philippe Blondeau. Arras : Artois Presses Université, 2010. 139-145.

CHOL, Isabelle. «Introduction ». LiVres de pOésie Jeux d'esPace. Ed. Isabelle Chol, Bénédicte Mathois et Serge Linarès. Paris : Honoré Champion, 2016. 9-26.

DEBON Claude et Martial LENGELLÉ. « Les enjeux du poème spatial : l'exemple de Pierre Garnier ». LiVres de pOésie Jeux d'esPace. Dir. Isabelle Chol, Bénédicte Mathois et Serge Linarès. Paris : Honoré Champion, 2016. 503-523.

DEPÉTRIS, Jean-Pierre, GARNIER, Ilse et Pierre GARNIER. « Bavardages épistolaires sur la poésie spatiale et sonore ». À travers champs 11 (automne 2001) : 4-12.

ÉDELINE, Francis. Pierre et Ilse Garnier. Liège : Yellow now, 1981.

ÉDELINE, Francis. « Ilse au pays des marelles. Réflexions sur quelques œuvres éphémères d'Ilse Garnier ». Deux poètes face au monde : Ilse et Pierre Garnier. Dir. Christine Dupouy. Tours : Presses universitaires François-Rabelais, 2018. 123-140.

ÉDELINE, Francis. « Introduction aux 'marelles du ciel' d'Ilse Garnier ». Association Ilse et Pierre Garnier, Infolettre 6 (avril 2019).

FONTANA, Giovanni. « Pierre Garnier et l'architecture ». Le temps des cathédrales dans l'œuvre d'Ilse et Pierre Garnier. Dir. Marianne Simon-Oikawa, à paraître.

GAPPMAYR, Gaby. Sprache und Raum - Die Poésie Spatiale von Pierre und Ilse Garnier. Bielefeld : Aisthesis Verlag, 2004.

GAPPMAYR, Gaby. « La poésie spatiale de Pierre et Ilse Garnier : le mystère de l'espace ». Pierre et Ilse Garnier : la poésie au carrefour des langues. Dir. Philippe Blondeau. Arras : Artois Presses Université, 2010. 71-76.

GARNIER, Ilse. « Fin du monde de l'expression ». Les Lettres 31 (4 e $^{\mathrm{e}}$ trimestre 1963) : 43-45.

GARNIER, Ilse. Blason du corps féminin. Paris : André Silvaire, 1979.

GARNIER, Ilse. rythmes et silence. Poèmes spatiaux. Paris : André Silvaire, 1980.

GARNIER, Ilse. Poème du i, Poème spatial. Paris : André Silvaire, 1981.

GARNIER, Ilse. Fensterbilder. Ein Stundenbuch. 1983. Calais : Atelier Xavier Hénicaux.

GARNIER, Ilse. Ermenonville, partition pour un promeneur solitaire (création d'un paysage sonore). Paris : André Silvaire, 1984.

GARNIER, Ilse. Quartett - ein Zahlentext. 1985. Archives familiales Ilse et Pierre Garnier.

GARNIER, Ilse. Puzzle-Alphabet, Jeu de Cubes. Liège : Éditions Quaternaire, 1988.

GARNIER, Ilse. Éros et Psyché. 1989. Archives familiales Ilse et Pierre Garnier.

GARNIER, Ilse. Eros und Psyché. 1989. Archives familiales Ilse et Pierre Garnier. 
GARNIER, Ilse. Éros et Psyché. Jazz pour les yeux. Anthologie de poésie spatiale. Paris : L'herbe qui tremble, 2011.

GARNIER, Ilse. La Meuse. Paris : André Silvaire, 1991.

GARNIER, Ilse. Poème cinématographique - scénario d'un poème visuel cosmique. 1993. Reproduit, avec une présentation de Violette Garnier, dans Deux poètes face au monde : Pierre et Ilse Garnier. Dir. Christine Dupouy. Tours : Presses Universitaires François-Rabelais, 2019. 193-201

GARNIER, Ilse. Winterlandschaft mit Vögeln [Paysage d'hiver aux oiseaux]. Lille : Alain Buyse, 1996 (réalisé en 1987-88-96).

GARNIER Ilse. Jazz pour les yeux. Anthologie de poésie spatiale. Paris : L'herbe qui tremble, 2011. GARNIER Ilse. chiendents, cahier d'arts et de littératures 22 (juin 2012).

GARNIER, Ilse et Pierre. Poèmes mécaniques. Paris : André Silvaire, 1965.

GARNIER, Ilse et Pierre. Prototypes. Textes pour une architecture. Paris : André Silvaire, 1965.

GARNIER, Ilse et Pierre. Othon III - Jeanne d'Arc. Structures historiques. Paris : André Silvaire, 1967.

GARNIER, Ilse et Pierre. Esquisses palatines. Paris : André Silvaire, 1971.

GARNIER, Ilse et Pierre. Jardin japonais. Paris : André Silvaire, 1977.

GARNIER, Ilse et Pierre. Poésie spatiale. Une anthologie. Préface d'Isabelle Maunet-Salliet. Marseille : Al Dante, 2012.

GARNIER, Pierre et Ilse. Japon. Ed. Marianne Simon-Oikawa. Paris : L'herbe qui tremble, 2 vols. 2016.

GARNIER, Pierre. « Hiver ». Les Lettres 29 ( $1^{\mathrm{er}}$ trimestre 1963).

GARNIER, Pierre. « Manifeste pour une poésie nouvelle, visuelle et phonique ». Les Lettres 29 (1 ${ }^{\text {er }}$ trimestre 1963$): 1-8$.

GARNIER, Pierre. « Deuxième manifeste pour une poésie visuelle ». Les Lettres 30 ( $2^{\mathrm{e}}$ trimestre 1963) : 15-28.

GARNIER, Pierre. « Poésie concrète. Panorama ». Les Lettres 33 (2e trimestre 1964) : 6-16.

GARNIER, Pierre. « Microstructures et microformats » a. Les Lettres 34 (4e trimestre 1965) : 28-29.

GARNIER, Pierre. « minipoems ». vers-univers (décembre 1966).

GARNIER, Pierre. I microcosmique. Toronto : Ganglia, 1967.

GARNIER, Pierre. « Microstructures et microformats » b. Spatialisme et poésie concrète. 1968. 87-106.

GARNIER, Pierre. « Position 4 du Spatialisme. Qu'est-ce que la micropoésie ? ", années 1960.

GARNIER, Pierre. Congo - Poème pygmée, poème spatial. Paris : André Silvaire, 1980.

GARNIER, Pierre. Tristan et Iseult (poème spatial). Paris : André Silvaire, 1981.

GARNIER, Pierre. Tintin's Reise in die Poesie [Le voyage de Tintin en poésie]. EIN KINDERBUCH poésie spatiale. Ed. k. Riha et s.j. Schmidt. Siegen : experimentelle texte 12, 1987.

GARNIER, Pierre. Lettre à Christian Janicot. 19 mai 1993. Fonds Anthologie du cinéma invisible. IMEC, carton 9, pochette 1 .

GARNIER, Pierre. Une mort toujours enceinte (une chronique), poèmes, tome 2. Amiens : Éditions Corps Puce, 1995. 
GARNIER, Pierre. 16 Gedichte in einem Quadrat [16 poèmes dans un carré]. Cologne : Édition Hundertmark, 1996.

GARNIER, Pierre. Depuis qu'il n'y a plus d'abeilles, la poésie a quitté le village. Gouzeaucourt : Le Corridor bleu, 1999.

GARNIER, Pierre. Poèmes sous microscope. Bordeaux : l'Attente, 1999.

GARNIER, Pierre. A vécu la disparition des bouvreuils. Marseille : Fidel Anthelme X, 2000.

GARNIER, Pierre. Les Constellations en 2002. Paris : Le Corridor bleu, 2003.

GARNIER, Pierre. L'isola. Ed. Julien Blaine, Jacques Donguy, Giovani Fontana, Patriozio Peterlini, Sarenco. Brescia : Fondazione Berardelli, 2007.

GARNIER, Pierre. Adolescence. Montreuil-sur-Brêche : Éditions des Vanneaux, 2008.

GARNIER, Pierre. Nanopoèmes. Achill Island : Rexfoxpress, 2011.

GARNIER, Pierre. Cuvres poétiques 1. 1950-1968. Montreuil-sur-Brêche : Éditions des Vanneaux, 2008.

GARNIER, Pierre. CEuvres poétiques 2. 1968-1988. Montreuil-sur-Brêche : Éditions des Vanneaux, 2009.

GARNIER, Pierre. CEuvres poétiques 3. 1979-2002. Montreuil-sur-Brêche : Éditions des Vanneaux, 2012.

GARNIER, Pierre et Seiichi NIIKUNI. Poèmes franco-japonais. Paris : André Silvaire, 1966.

GARNIER, Violette. Ilse Garnier - Voyage cosmique 1986-1996. Deux poètes face au monde : Ilse et Pierre Garnier. Dir. Christine Dupouy. Tours : Presses universitaires François-Rabelais, 2018. 193-201.

KÜHN, Renate. Pierre et Ilse Garnier : la poésie au carrefour des langues. Dir. Philippe Blondeau. Arras : Artois Presses Université, 2010. 93-103.

LENGELLÉ, Martial. L'œuvre poétique de Pierre Garnier. Angers : Presses de l'Université d'Angers, 2001.

MARTINEZ, Meritxell et Albert COMA. « Poème cinématographique ». 2016. https://vimeo.com/ 183284925 (page consultée le 28 mars 2021).

MAUNET-SALLIET, Isabelle. « Poétique du passage. Lecture de Leonardo (1998) d'Ilse Garnier ». Deux poètes face au monde: Ilse et Pierre Garnier. Dir. Christine Dupouy. Tours : Presses universitaires François-Rabelais, 2018. 39-50.

MEEGLIN-DELCROIX, Anne. Esthétique du livre d'artiste. Paris : Éditions Jean-Michel Place, 1997. SIMON-OIKAWA, Marianne. « Ilse Garnier et le livre d'artiste : Matière et construction poétique dans Winterlandschaft mit Vögeln ». Francophonie vivante 2 (2019a) : 126-133.

SIMON-OIKAWA, Marianne. Les Poètes spatialistes et le cinéma. Paris : Nouvelles éditions Place, $2019 b$.

SIMON-OIKAWA, Marianne. «Esthétique de la "poésie habitable”. Prototypes / Textes pour une architecture d'Ilse et Pierre Garnier ». Le temps des cathédrales dans l'œuvre d'Ilse et Pierre Garnier. Dir. Marianne Simon-Oikawa, à paraître.

SOUCHIER, Emmanuël. « Histoires de pages et pages d'histoire ». L'Aventure des écritures. La page. Dir. Anne Zali, 1999. 19-55. 
ZERBIB, David. « Introduction. Voulez-vous enregistrer les modifications? ». In Octavo. Des formats de l'art. Dir. David Zerbib. Annecy ; Dijon : ESAAA Éditions / les Presses du Réel, 2015. 13-19.

\section{NOTES}

1. Voir I. et P. Garnier, Poésie spatiale, une anthologie (2012) ; I. Garnier, Jazz pour les yeux. Anthologie de poésie spatiale (2011) ; P. Garnier, Euvres poétiques (2008-2012) ; SimonOikawa, Les Poètes spatialistes et le cinéma (2019b).

2. Voir Édeline (1981), Lengellé (2001, 127-271), Gappmayr (2004 et 2010), Kühn (2010), Buschinger (2021), Debon et Lengellé (2016).

3. Cette scène est en réalité le souvenir à peine transposé d'une histoire vraie, rapportée à Pierre Garnier par le peintre Roger Toulouse dans les années 1980. Un jour de 1942, étant descendu voir Max Jacob dans la pièce du premier étage où il l'hébergeait, Toulouse trouva le poète occupé à travailler. L'auteur du Cornet à dés avait, étalée devant lui, une carte du ciel dont il reportait les différentes étoiles sur une feuille de papier Canson posée à côté, en leur donnant de nouveaux noms (Entretien avec l'auteur, 4 avril 1999).

4. Le concept de constellation, emprunté au poète Eugen Gomringer qui l'avait luimême trouvée chez Mallarmé, est essentiel dans la poésie concrète. Dans un article intitulé "Poésie concrète. Panorama », Pierre Garnier cite la définition qu'en donne Gomringer lui-même : «La constellation est un ordre et en même temps un espace de jeu avec des grandeurs fixes. La constellation est fixée par le poète qui détermine l'espace, le champ des forces et désigne ses possibilités » (8). Pierre Garnier publiera lui-même un recueil de poèmes intitulé Les Constellations en 2002.

5. Dossier génétique conservé dans les archives familiales Ilse et Pierre Garnier. Le dossier comprend des brouillons de la préface, des notes de travail, ainsi que des poèmes dont tous n'ont pas été repris dans le recueil publié.

6. Jean-Pierre Depétris avait bien senti l'importance du mouvement dans les livres spatialistes. Dans son entretien avec Ilse et Pierre Garnier, il remarque: "Jusqu'à maintenant, à ma connaissance, on a surtout parlé du poème spatialiste au sein de l'espace de sa page. Mais les pages se suivent au sein de livres ou de cahiers. Ils ne sont pas de simples recueils de textes autonomes. Ce qui se passe dans la suite des pages est au moins aussi intéressant que ce qui se passe au sein de chacune. À la place qu'y occupe le mot, la suite des pages ajoute peut-être le mouvement " (Depétris, I. et P. Garnier 10).

7. Ilse Garnier, enregistrement sonore d'un entretien inédit avec Lydie Prioul, archives familiales Ilse et Pierre Garnier.

8. Violette Garnier, Jacques Donguy, Thierry Chauveau et Gaby Gappmayr ont bien voulu m'apporter de précieux détails sur les différentes versions connues de cette œuvre. Qu'ils en soient ici vivement remerciés.

9. Ces « ciné-poèmes » n'aboutirent pas à un film réalisé par Ilse Garnier ou avec sa collaboration, mais une interprétation en fut donnée sous forme de vidéo par Meritxell Martinez et Albert Coma (2016) : https://vimeo.com/183284925 (consulté le 28 mars 2021). 


\section{RÉSUMÉS}

Figures des avant-gardes et inventeurs du «spatialisme» au début des années soixante, Ilse Garnier (1927-2020) et Pierre Garnier (1928-2014) sont surtout connus pour leurs créations dans le domaine de la poésie visuelle et de la poésie sonore. Leurs œuvres investissent une grande variété de supports (papier, bande magnétique, pellicule photographique), de formats (depuis des objets tenant dans la main à d'autres mesurant un mètre de côté) et de dispositifs (feuilles séparées, livres reliés, projection de diapositives, installations éphémères). Cette diversité se comprend par rapport aux principes même de leur poésie, où l'espace joue un rôle central. L'article montre comment dans leurs œuvres visuelles les poètes ont joué avec les supports et déjoué les codes, n'hésitant pas chaque fois que possible à faire sortir le poème de la page, à renouveler les formes du livre, et à faire circuler leurs créations d'un support à un autre.

Prominent representatives of the avant-gardes and inventors of "spatialism" in the early sixties, Ilse Garnier (1927-2020) and Pierre Garnier (1928-2014) are best known for their creations in the field of visual poetry and sound poetry. Their works use a large variety of media (paper, tape, photographic film), formats (from objects that can be held in the hand to others measuring a meter across) and objects (separate sheets, bound books, slides, ephemeral installations). This diversity is linked to the very principles of their poetry, where space plays a central role. The article examines how in their visual works the poets played with supports and thwarted codes, not hesitating whenever possible to let the poem expand out of the page, to renew the forms of the book, and to circulate their creations from one medium to another.

\section{INDEX}

Mots-clés : Garnier (Ilse), Garnier (Pierre), spatialisme, poésie visuelle, espace, page, livre, diapositive, photographie, livre d'artiste

Keywords : Garnier (Ilse), Garnier (Pierre), spatialism, visual poetry, space, page, book, slide, photography, artist's book

\section{AUTEUR}

\section{MARIANNE SIMON-OIKAWA}

Université de Tokyo

Marianne Simon-Oikawa est Maîtresse de conférences HDR à l'Université de Tokyo, chercheuse associée à l'Institut français de recherche sur le Japon (UMIFRE 19) de la Maison franco-japonaise (Tokyo), et à l'Université Paris 3 (équipe «Écritures de la modernité », UMR THALIM). Ses travaux portent sur les relations entre le texte et l'image en France et au Japon. Dans le domaine de la poésie visuelle, elle a consacré de nombreux travaux à Pierre Albert-Birot (codirection avec Carole Aurouet de Pierre Albert-Birot, 1876-1967. Un pyrogène des avant-gardes, Paris, Presses universitaires de Rennes, coll. «Interférences », 2019, et de Poésie vivante - Hommage offert à Arlette Albert-Birot, coll. « Poétiques et esthétiques XXe-XXIe siècle », Paris, Champion, 2012, 384 p.). Depuis plusieurs années, elle travaille plus particulièrement sur l'œuvre d'Ilse et Pierre Garnier. Elle a publié notamment Pierre et Ilse Garnier, Japon (textes choisis, établis et présentés par Marianne Simon-Oikawa, 2 vols.), L'herbe qui tremble (2016), et Les Poètes spatialistes et le cinéma (Paris, Nouvelles éditions Place, coll. « Le cinéma des poètes », 2019). Elle prépare 
actuellement les actes du colloque Le temps des cathédrales dans l'œuvre d'Ilse et Pierre Garnier (mai 2021), à paraître. 